\title{
OPTIMIZATION OF INTERSECTION CONTROL SCHEME CONSIDERING PHASE-MOVEMENT-COMBINATION UNDER AUTOMATED VEHICLES ENVIRONMENT
}

\author{
Wenbin XIAO ${ }^{1}$, Shunying $\mathrm{ZHU}^{2 *}$, Daobin $\mathrm{WANG}^{3}$, Wei $\mathrm{LIU}^{4}$ \\ 1,2School of Transportation, Wuhan University of Technology, China \\ ${ }^{3}$ School of Automobile and Traffic Engineering, Wuhan University of Science and Technology, China \\ ${ }^{4}$ College of Traffic and Transportation, Chongqing Jiaotong University, China
}

Submitted 16 November 2018; resubmitted 30 April 2019; accepted 28 September 2019;

first published online 19 August 2020

\begin{abstract}
For signal control intersection, the Phase-Movement-Combination (PMC) styles could directly impact the control performance of the signal scheme. Automated vehicles use mechatronics technology to drive autonomously and safely according to the predetermined lane trajectory, which caused the phase movement combination and Phase Combination (PC) schemes become more and more complicated. Therefore, this paper proposed a method to consider the extensive PMC styles by fractionalizing movement compatibility relationships, and used discrete mathematics to calculate overall Feasible Phase Combination (FPC) schemes according to the requirements of the signal phase. A corresponding optimal timing model was also established for FPC schemes by minimizing the average vehicle delay and maximizing the intersection capacity. Results were compared against the conventional PC schemes for a variety of demand scenarios. It was concluded that the proposed signal control optimization method was effective to optimize the intersection control scheme, depending on different demand scenarios.
\end{abstract}

Keywords: intersection signal control, movement compatibility, phase-movement-combination, phase combination scheme, timing optimization model, automated vehicles.

\section{Notations}

To facilitate model presentation, notations used hereafter are listed in the running text.

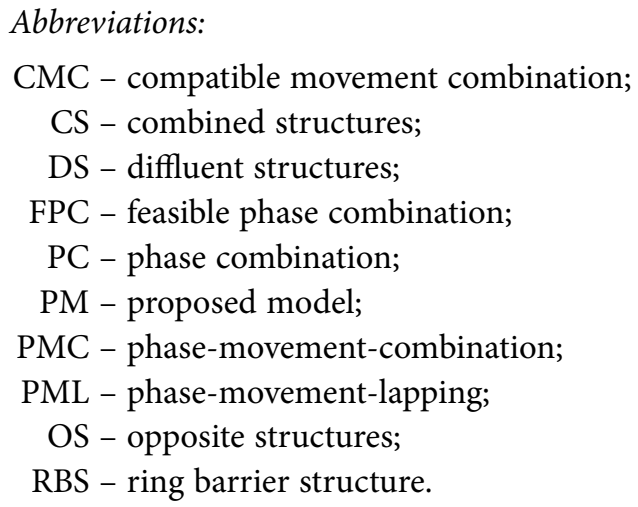

CMC - compatible movement combination;

CS - combined structures;

DS - diffluent structures;

FPC - feasible phase combination;

$\mathrm{PC}$ - phase combination;

PM - proposed model;

PMC - phase-movement-combination;

PML - phase-movement-lapping;

OS - opposite structures;

RBS - ring barrier structure.

Variables and definitions:

$A$ - the yellow time;

$A D^{w}$ - the average delay of the entire intersection for $\mathbf{F P C}_{w}$;

$A D_{i}^{w}$ - the average delay of approach $i$ for $\mathbf{F P C}_{w}$;

$A D_{i j}^{w}$ - the movement $\sigma_{i j}$ average delay of the $\mathbf{F P C}_{w}$;

$A D_{i j}^{w, 1}$ - the uniform delay;

$A D_{i j}^{w, 2}$ - the incremental delay;

$A D_{i j}^{w, 3}$ - the initial queue delay, if no queue exists at the end of the analysis period, the value is 0 ;

$C^{w}$ - the cycle time length of the $\mathbf{F P C}_{w}$;

$C_{\min }, C_{\max }$ - the minimum and maximal cycle time length;

CCM - the set of the combination of confluence movements;

$\mathbf{C C M}_{\gamma}$ - the movement set of confluence relation;

$\mathrm{Ccm}_{\gamma}$ - describe the compatibility relation of movements in $\mathbf{C C M}_{\gamma}\left(\mathrm{Ccm}_{\gamma}=0\right.$ or 1$)$;

*Corresponding author. E-mail: zhusy2001@163.com 
CDM - the set of all combinations of diffluence movements;

$\mathrm{CDM}_{\alpha}$ - the movement combination of diffluence relation;

$C d m_{\alpha}$ - describe the compatibility relation of movements in $\mathbf{C D M}_{\alpha}\left(C d m_{\alpha}=0\right.$ or 1$)$;

$C L G^{w}$ - the capacity of the entire intersection for $\mathbf{F P C}_{w}$;

$C L G_{i}^{w}$ - the capacity of approach $i$ for $\mathbf{F P C}_{w}$;

$C L G_{i j}^{w}$ - lane group capacity;

COM - the set of the combination of opposite movements;

$\mathrm{COM}_{\beta}$ - the movement combination of opposite relation;

$\mathrm{Com}_{\beta}$ - describe the compatibility relation of movements in $\mathbf{C O M}_{\beta}\left(\mathrm{Com}_{\beta}=0\right.$ or 1$)$;

CMC - the set of the combination of compatible movements;

$e_{i j}$ - the binary variables, represents the presence of the movement $\sigma_{i j}\left(e_{i j}=0\right.$ or 1$)$;

$f$ - the upstream filtering adjustment factor, and for an isolated intersection (its value is 1.0);

$\mathbf{F P C}_{w}$ - the $w$ th FPC scheme;

$G_{i j}^{w}$ - the green time of movement $\sigma_{i j}$ in $\mathbf{F P C}_{w}$;

$G_{\min }, G_{\max }$ - the minimum and maximal green time; $G e_{i \mid e_{i 2}=1}^{w}$ - the effective green time of the approach $i$

$G e_{i j}^{w}$ - the movement $\sigma_{i j}$ effective green time of the FPC $_{w}$;

$G e_{i j}^{w}$ - the effective green time of movement $\sigma_{i j}$ in $\mathbf{F P C}_{w}$;

$G e p_{i j}^{w} l_{i j}^{w, k}$ - the effective green time of phase $l_{i j}^{w, k}$ for movement $\sigma_{i j}$ in $\mathbf{F P C}_{w}$;

$G p_{i j}^{w}{ }_{i j}^{w, k}$ - the green time of phase $l_{i j}^{w, k}$ for movement $\sigma_{i j}$ in $\mathbf{F P C}_{w}$;

$i$ - intersection approach $(i=1,2,3,4)$;

$k, k^{\prime}$ - the ordinal order of the phases that contain movement $\sigma_{i j}$;

$l_{i j}^{w, k}$ - the identifier of the phase that contains movement $\sigma_{i j}$ in $\mathbf{F P C}_{w}\left(l_{i j}^{w, k}=x_{i j}^{w, k}+1\right)$;

$m_{w}$ - the phase number of the corresponding FPC scheme;

$m_{v}$ - the phase number of the corresponding PC scheme;

MSI - movements' set of intersection;

$\mathrm{PC}_{v}$ - the vth PC scheme;

$r$ - the incremental delay factor, which for pretimed signal control is 0.5 ;

$T_{a p}$ - the analysis period duration (generally $0.25 \mathrm{~h}$ );

$T^{w, u}$ - the time length of $u$ th phase in $\mathbf{F P C}_{w}$;

$T_{i j}^{w} l_{i j}^{w, k}$ - the time length of phase $l_{i j}^{w, k}$ in $\mathbf{F P C}_{w}$ $\left(T_{i j}^{w, l_{i j}^{w, k}}=T^{w, l_{i j}^{w, k}}\right)$;

$w$ - the number of the FPC scheme; $x_{i j}^{w, k}$ - the identifier of the element that contained movement $\sigma_{i j}$ in $\operatorname{Charfp} c_{w}\left[m_{w}\right]$;

$\delta, \psi, \varphi$ - the element identifiers of the array Char $p c_{v}\left[m_{v}\right], \delta, \psi, \varphi \in N$;

$\eta_{i j}^{w}$ - the number of elements that contained movement $\sigma_{i j}$ in Char fpc $c_{w}\left[m_{w}\right]$;

$\sigma_{i j}$ - intersection movement;

$\tau$ - non-zero natural number $\left(\tau \in N^{+}\right)$;

$v$ - the identifier of the PC scheme;

$\xi_{i j}^{w}$ - the movement $\sigma_{i j}$ volume-to-capacity ratio of the $\mathbf{F P C}_{w}$.

\section{Introduction}

With the rapidly growing urban traffic demand, congestion and other traffic problems occur more and more frequently. Reasonable measures to alleviate urban traffic problem have been recently addressed. Signalized intersections have been proved as the bottlenecks in urban traffic networks. Signal control optimization at signalized intersections can effectively reduce urban traffic congestion and improve traffic efficiency. Currently, urban traffic control strategies tend to focus on the control of individual intersections (Gayah, Daganzo 2012; Guler, Cassidy 2012; Liu et al. 2009). There are three general approaches for the intersection control, namely the stage-based method, lane assignment, modification design. The stage-based method mainly focuses on how to establish objective functions by selected different indexes: delay (Fu, Hellinga 2000; Ghanbarikarekani et al. 2018; He et al. 2014; Sun et al. 2006; Zheng et al. 2010), traffic capacity (Chang, Sun 2004), number of stops (Lucas et al. 2000), queue length (Comert 2016; Liu et al. 2017; Mung et al. 1996; Zhao et al. 2015b) or combinatorial optimization of multiple indexes (Akçelik, Rouphail 1993; Li et al. 2013), to optimize signal control scheme. For lane assignment method, Wong, C. K., Wong, S. C. (2003) and Wong, Heydecker (2011) present a lane-based setting method for the integrated design of lane markings and signal settings for isolated intersection, and introduce the setting requirements for different movements in the same approach. Then they built the corresponding objective function to determine the optimal lane markings and signal settings. For modification design of intersection, some researches adopted various innovative intersection designs in their effort to enhance the intersection capacity, such as the median U-turn intersection, jug-handle, superstreet intersection, and so on (Goldblatt et al. 1994; Hummer 1998a, 1998b; Suh, Hunter 2014; Xuan et al. 2011; Zhao et al. 2013, 2015a). These designs changed the conflict area of partial movements at the intersection or simplified the internal movement layout of primary intersection; so that the left-turn flow and the through movement (or the through movement on the adjacent leg) can progress concurrently, thereby increased the capacity of the intersection. Furthermore, Xuan et al. (2011) proposed that, a separate turn phase is often used on the approach leg to an intersection with 
heavy left turns. This wastes capacity on the approach because some lanes cannot discharge the vehicles during the green time. Therefore, they proposed a tandem intersection design to sort the left-turn movement and through movement in the sorting area by using a pre-signal. However, the disadvantage is that the tandem sorting area has to be long enough to accommodate the vehicles in queue. Yang and Shi (2017) proposed a modified sorting strategy based on the main signal phase swap in order to reduce the length requirement for the sorting area and improve the operability.

All the methods discussed above mainly focus on optimizing signal timing and modifying approach layout. For the signal control at urban road intersections, another important factor is PC scheme, which was rarely mentioned in previous researches. For intersection movements, there are compatible and conflict relation according to the position of the movement in the approach. The so-called movement compatibility relation is that there is no cross point between two movements. According to the right rules, the left turn lane is located on the left side of the through lane. The compatible relation of movements includes the opposite compatible, diffluence compatible, and confluence compatible. The opposite compatible indicates that the movements are non-conflict, and locate in the opposite approach of intersection. Similarly, the diffluence compatible indicates that the movements are non-conflict, and locate in the same approach of intersection. The confluence compatible indicates that the movements are nonconflict relation, locate in different approach of intersection, and they flow into the same exit of intersection.

At present, the four-PC scheme is often used to obtain the PC scheme, and it mainly includes OS, DS and CS. The so-called OS is that every phase is composed of movements, which are opposite compatible relation, the DS is that every phase is composed of movements, which are diffluence compatible relation, and the CS is the mixture of DS and CS. The specific structural styles were shown in Table 1.

In general, the queue lengths of different movements are different, even in a same phase. When the control scheme adopted four-PC style, and a signal phase turned into green, the vehicles in a long queue movement would pass through the intersection at a saturated flow rate, but the vehicles of another movement of same phase would pass through the intersection at an arrival rate. The arrival rate of the movement is less than the saturation flow rate for the unsaturated condition. Therefore, the case can result in the loss of green time of that phase.

In order to reduce the loss of green time of phase, PML was considered here, which is that one movement crosses two or more adjacent phases. At present, the RBS (Figure 1) is often used to generate PC scheme of signal intersection, which well-considered the PML (Feng et al. 2015).

It can be found from Figure 1 that the RBS includes all four-PC schemes, then perfectly combines the diffluence and opposite compatible relation of the movements,
Table 1. Traditional four-PC scheme

\begin{tabular}{|c|c|c|c|c|}
\hline \multirow{2}{*}{ Style } & \multicolumn{4}{|c|}{ Phase scheme } \\
\hline & Phase 1 & Phase 2 & Phase 3 & Phase 4 \\
\hline OS & 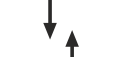 & & & \\
\hline DS & $\longleftarrow$ & & $\underset{\longrightarrow}{\longrightarrow}$ & \\
\hline CS & $\longleftarrow$ & $\underset{\sim}{\longrightarrow}$ & & \\
\hline
\end{tabular}

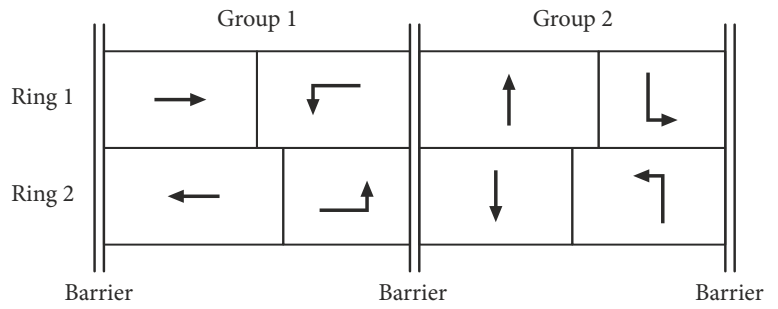

Figure 1. RBS

achieves flexible lapping for different phases. For unautomated vehicles environment, the vehicles of confluence relation movement flowing into the same exit of intersection may lead to potential traffic problems, so the RBS did not consider the confluence compatible. However, for automated vehicle, it can depend on mechatronics technology to drive autonomously and safely according to the predetermined lane trajectory ( $\mathrm{Li}$ et al. 2014); the confluence relation between movements may consider to be compatible relation under some cases. Therefore, the PC scheme obtained by RBS would not be comprehensive.

The current researches on signal optimization for automatic vehicle mainly focus on vehicle data collection and automatic driving behaviour at intersection (Jamson et al. 2013; Li et al. 2014), but seldom focus on PC scheme. However, with joining confluence compatibility combination of movement, the researches on PC schemes will be thriving. In urban road network, the traffic flow of each intersection is stochastic, and the main movements are quite different between each other, and the current methods only considered two kinds of movement combination styles of phase, and cannot get the comprehensive PC schemes under automated vehicles environment. Therefore, it is necessary to design a new optimization method of signal control scheme to match the random arrivals under automated vehicles environment.

In this concern, this paper fractionized three kinds of movement compatibility relations and fully considered the PMC, studied the PMC styles, used discrete mathematics to calculate overall FPC schemes. Finally, the corresponding optimal timing model was established to obtain an optimal control scheme.

The remainder of this paper was arranged as follows. Section 1 presented CMC. Section 2 studied PC scheme. 
Section 3 built a signal phase optimization-timing model. Evaluation of the PM performance was conducted in Section 4 through extensive numerical and simulation analyses. Conclusions and further work were given in last section.

\section{CMC under automated vehicles environment}

\subsection{Compatibility relation of movements}

For intersection, the movement $\sigma_{i j}(i=1,2,3,4 ; j=1,2,3)$ symbol of every approach $i$ were showed in Figure 2. In general, the intersection sets exclusive right-turn lanes by channelization for right turn movement according to the right rules, so right turn movement was not considered in this paper. When $j=2, \sigma_{i 2}$ was the movement generated by a shared lane that represented a movement of two directions; other symbols represented movement of a single direction.

Discrete mathematics was used to research the CMC. The intersection may not include all movements in Figure $2, e_{i j}$ represents if a movement is included in the movement set of intersection MSI:

$$
e_{i j}= \begin{cases}0, & \sigma_{i j} \notin \text { MSI; } \\ 1, & \sigma_{i j} \in \text { MSI. }\end{cases}
$$

The relation between movements included the diffluence, opposite and confluence relation. Thus, the set of all combinations of diffluence movements can be set as:

$$
\mathbf{C D M}=\left\{\mathbf{C D M}_{\alpha}, \alpha \in N^{+}\right\},
$$

where: $\alpha$ is an approach of the intersection, $\alpha=1,2,3,4$. In addition, the movement combination of diffluence relation can be represented as:

$$
\mathbf{C D M}_{\alpha}=\left\{\sigma_{\alpha j} \mid \sigma_{\alpha j} \in \mathbf{M S I}\right\} .
$$

To better describe the compatibility relation of movements in $\mathbf{C D M}_{\alpha}$, the $C d m_{\alpha}$ parameter was introduced as follows:

$$
C d m_{\alpha}= \begin{cases}0, & \text { incompatible; } \\ 1, & \text { compatible }\end{cases}
$$

when the movements in $\mathbf{C D M}_{\alpha}$ are compatible: $C d m_{\alpha}=1$, otherwise $C d m_{\alpha}=0$. According to the definition of the movement compatibility relation, all movements in $\mathbf{C D M}_{\alpha}$ were compatible when the left turn lane was located on the left side of the through lane. Thus, $C d m_{\alpha} \equiv 1, \forall \alpha$.

The opposite relation of movements was the relationship between two opposite movements, which were located in the two contrary approaches of intersection. COM was defined as the set of the combination of opposite movements:

$$
\mathbf{C O M}=\left\{\operatorname{COM}_{\beta}, \beta \in N^{+}\right\} .
$$

In general, the $\mathbf{C O M}_{\beta}$ are considered as the combination of opposite movements. However, when a $\mathbf{C O M}_{\beta}$

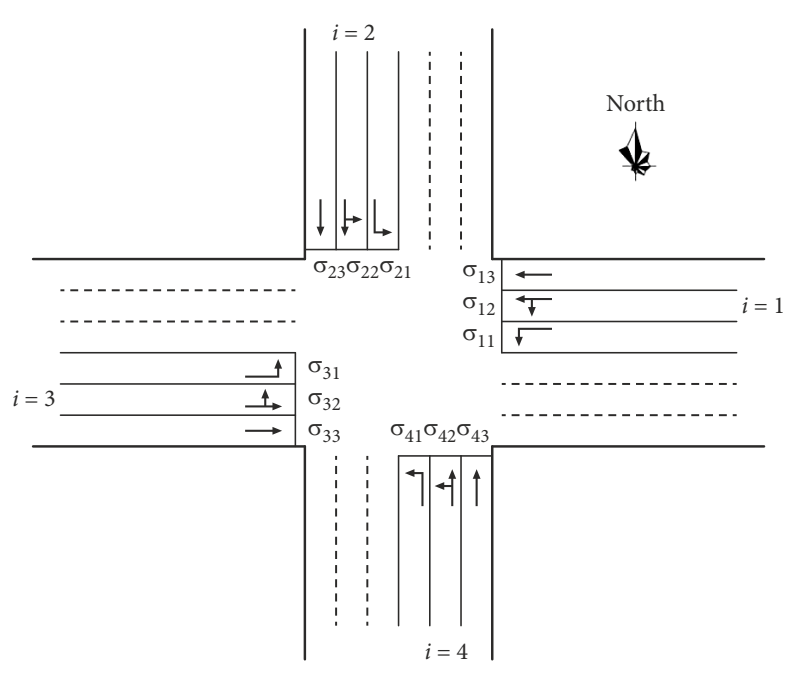

Figure 2. Movement symbols

movement shared a lane with another movement, the $\mathrm{COM}_{\beta}$ were considered as an incompatible relation. Therefore, the $\mathrm{Com}_{\beta}$ parameter was introduced as follows:

$$
\operatorname{Com}_{\beta}=\left\{\begin{array}{ll}
0, & \sigma_{i j \mid e_{i 2}=1} \in \mathbf{C O M}_{\beta} ; \\
1, & \sigma_{i j \mid e_{i 2}=1} \notin \mathbf{C O M}_{\beta} ;
\end{array} ; j,\right.
$$

where: $\sigma_{i j e_{i 2}=1}$ is a movement in approach $i$ with shared lane, for example, if the approach 1 had the shared lane $\left(i=1, e_{12}=1\right)$, so $\sigma_{1 j \mid e_{12}=1}=\sigma_{1 j}$. And when $\mathbf{C O M}_{\beta}$ do not include the movement $\sigma_{i j \mid e_{i 2}=1}$, the combination $\mathbf{C O M}_{\beta}$ are compatible, and $\operatorname{Com}_{\beta}=1$.

Finally, a confluence relation existed between movements, which was the relationship between two movements located in the two neighbouring approaches of the intersection. The two movements flowed into the same intersection exit. Thus, CCM was defined as a set of the combination of confluence movements:

$$
\mathbf{C C M}=\left\{\mathbf{C C M}_{\gamma}, \gamma \in N^{+}\right\} \text {. }
$$

Likewise, to describe the compatibility of movements in $\mathbf{C C M}_{\gamma}$, the $\mathrm{Ccm}_{\gamma}$ parameter was introduced as follows:

$$
C c m_{\gamma}= \begin{cases}0, & \text { incompatible; } \\ 1, & \text { compatible, }\end{cases}
$$

when the movements in $\mathbf{C C M}_{\gamma}$ are compatible: $\mathrm{Ccm}_{\gamma}=1$, otherwise $\mathrm{Ccm}_{\gamma}=0$.

At present, the confluence relationship of movements is considered as a conflict relationship under unautomated vehicles environment, so $\mathrm{Ccm}_{\gamma} \equiv 0$. However, in the automated vehicles environment, all vehicles can use mechatronics to autonomously perform driving functions and drive safely according to the predetermined lane trajectory, so the movement compatibility of confluence relation should be fractionized. If $\sigma_{i j \mid e_{i 2}=1} \in \mathbf{C C M}_{\gamma}$, then $\mathrm{Ccm}_{\gamma}=0, \forall \gamma$. But, if $\sigma_{i j \mid e_{i 2}=1} \notin \mathbf{C C M}$, the two movements in $\mathbf{C C M}_{\gamma}$ may be compatible, and other factors should be considered. 
If the two movements in $\mathbf{C C M}_{\gamma}$ were compatible, the following two conditions must be satisfied at the same time. Condition (1): The movements in $\mathbf{C C M}_{\gamma}$ did not share lanes with other movements. Condition (2): The total lane number occupied by two confluence movements at intersection should match with the lane number of the corresponding exit.

For condition (1):

$$
\sigma_{i j \mid e_{i 2}=1} \notin \mathbf{C C M}_{\gamma} \text {. }
$$

For condition (2), it was assumed that the automate vehicles pass through the intersection according to the predetermined lane trajectory. Therefore, Equation (10) must be satisfied:

$$
N_{\mathrm{CCM}_{\gamma}(1)}+N_{\mathrm{CCM}_{\gamma}(2)} \leq N_{\gamma},
$$

where: $N_{\mathrm{CCM}_{\gamma}(1)}, N_{\mathrm{CCM}_{\gamma}(2)}$ are the occupied lane numbers of movements (elements) in $\mathbf{C C M}_{\gamma} ; N_{\gamma}$ is the lane number of the corresponding exit $\gamma$ of movements in $\mathbf{C C M}_{\gamma}$.

To better understand condition (2), the following descriptions in Figure 3 were presented. Assuming two movements $\sigma_{\alpha}$ and $\sigma_{b}$ were confluence relation, $N_{\sigma_{a}}$ and $N_{\sigma_{b}}$ were the lane numbers of movements $\sigma_{\alpha}$ and $\sigma_{b}$, respectively, and $N_{a \& b}$ was the number of two movements $\left(\sigma_{a}\right.$ and $\left.\sigma_{b}\right)$ corresponding to the exit lane. In Figure $3 a$ $N_{\sigma_{a}}=1, N_{\sigma_{b}}=1, N_{a \& b}=3$, and $N_{\sigma_{a}}+N_{\sigma_{b}}<N_{a \& b}$, so $\sigma_{\alpha}$ and $\sigma_{b}$ were compatible according to Equation (10). In Figure 3b $N_{\sigma_{a}}=2, N_{\sigma_{b}}=1, N_{a \& b}=3$, and $N_{\sigma_{a}}+N_{\sigma_{b}}=N_{a \& b}$, so $\sigma_{\alpha}$ and $\sigma_{b}$ were also compatible according to Equation (10). In Figure $3 c N_{\sigma_{a}}=3, N_{\sigma_{b}}=1, N_{a \& b}=3$, and $N_{\sigma_{a}}+N_{\sigma_{b}}>N_{a \& b}$, therefore, there was a conflict point between $\sigma_{\alpha}$ and $\sigma_{b} ; \sigma_{\alpha}$ and $\sigma_{b}$ were incompatible.

Thus, if movements in $\mathbf{C C M}_{\gamma}$ were compatible, Equation (11) should be satisfied, and $C c m_{\gamma}=1$ :

$$
\left\{\begin{array}{l}
\sigma_{i j \mid e_{i 2}=1} \notin \mathbf{C C M}_{\gamma} ; \\
N_{C C M_{\gamma}(1)}+N_{C C M_{\gamma}(2)} \leq N_{\gamma} .
\end{array}\right.
$$

\subsection{CMC}

Therefore, CMC can be obtained by judging the compatibility of the movements, such as:

$$
\begin{aligned}
& \mathbf{C M C}=\left\{\mathbf{C D M}_{\alpha \mid C d m_{\alpha}=1}\right\} \cup \\
& \left\{\mathbf{C O M}_{\beta \mid \operatorname{Com}_{\beta}=1}\right\} \cup\left\{\mathbf{C C M}_{\gamma \mid C c m_{\gamma}=1}\right\},
\end{aligned}
$$

where: $\mathbf{C D M}_{\alpha \mid C d m_{\alpha}=1}$ is the set of the $C d m_{\alpha}=1$ corresponding to $\mathbf{C D M}_{\alpha} ; \mathbf{C O M}_{\beta \mid \mathrm{Com}_{\beta}=1}$ and $\mathbf{C C M}_{\gamma \mid C \mathrm{C} m_{\gamma}=1}$ are similar.

To help understand, the determination process of set CMC was described in Figure 4.

The purpose of this section was to facilitate the research of the next chapter, which discussed the signal PC scheme.
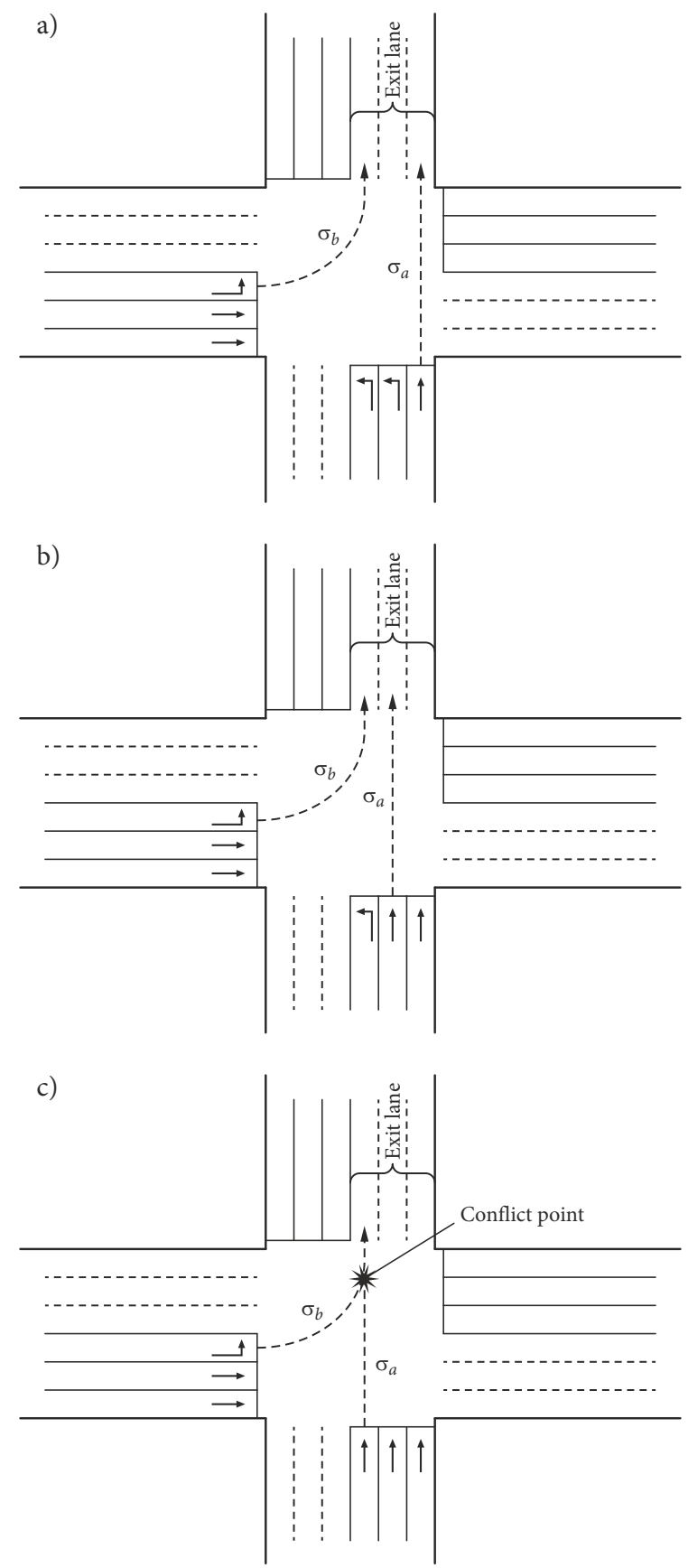

Figure 3. Compatibility of confluent movements: a - compatibility relation; b - compatibility relation; c - conflict relation

\section{Signal PC scheme}

Intersection signal control was determined by setting up different signals phases to make each conflict movement vehicle through the intersection at different time periods and to avoid vehicles meeting in an intersection collision zone. To ensure traffic safety, this paper set the protection phase for the left-turn movements of all approaches. Therefore, the movements of one phase must meet the non-conflict requirements. The compatibility of movements indicates that there is no point of conflict between movements. 


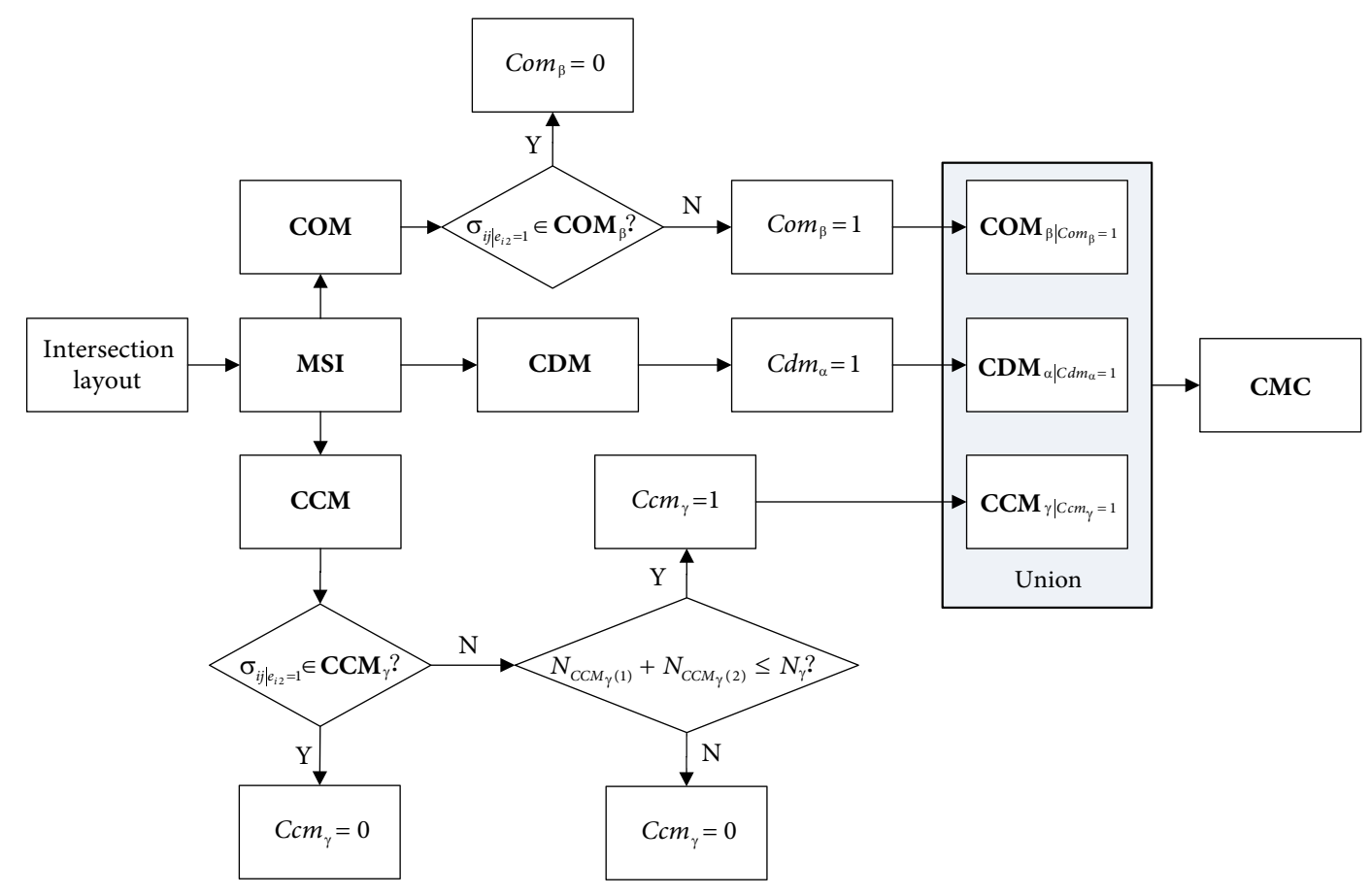

Figure 4. Determination process of set CMC

It was consistent with the setting requirements of the signal phase, so each CMC could be used as a signal phase, and the number of usable signal phases at intersections was the number of CMC elements.

To determine the FPC scheme, the PC scheme must be obtained firstly. The so-called PC scheme was composed of some usable signal phases arranged in a certain order. The elements in the set have not sequence characteristics, and for the PC scheme of the signal intersections, its setting had a phase sequence requirement. The different phase sequence setting had a significant influence on the control scheme, so the set cannot be used to describe the signal PC scheme. In this paper, an array was introduced to describe the PC scheme of the intersection signal control, and it was the set of elements of the same data type in a certain order.

The number of elements in set CMC represented the usable signal phase number of intersections, and a series of PC schemes can be obtained by randomly sampling each element. Each PC scheme was expressed by the character array, namely $\operatorname{Char}_{p} c_{\mathrm{v}}\left[m_{\mathrm{v}}\right]$, and:

$$
\begin{aligned}
& P C_{v}=\left\{C h a r p c_{v}\left[m_{v}\right],\right. \\
& \left.v \in N^{+} \text {and } m_{v} \in N^{+}\right\},
\end{aligned}
$$

where: $v$ is the identifier of the PC scheme; $m_{v}$ is the phase number of the corresponding PC scheme, their maximum values are respectively $\sum_{\tau=1}^{|\mathrm{CMC}|} C_{|\mathbf{C M C}|}^{\tau} A_{\tau}^{\tau}, \tau \in \quad$ and $|\mathbf{C M C}|$.

After all, signal PC schemes were determined, not every PC scheme can satisfy the phase setting requirements of the signal intersection. The PC schemes were feasible
(FPC), which must meet the phases setting requirements of control scheme. The specific setting requirements include two conditions: (1) the PC scheme must contain all movements of intersection; (2) if the same movement took up multiple phases (i.e., the lapping movement), these phases must be adjacent to each other. The setting of this condition ensured the continuous release of movements during a cycle, and reduced the loosing time of movement vehicles.

Condition (1):

$\bigcup_{\delta=0}^{m_{v}-1} p c_{v}[\delta]=$ MSI,$\delta \in N$.

Condition (2):

The array $C h a r p c_{v}\left[m_{v}\right]$ with 2 situations satisfied condition (2). Defined $\psi$ and $\varphi$ were the element identifiers of Char $p c_{v}\left[m_{v}\right]$, and $\psi>\varphi$. So $\psi, \varphi \in N$, and $\psi$, $\varphi \in\left[0, m_{\mathrm{v}}-1\right]$ :

1) there was no common movement between other elements except that the adjacent elements:

$$
\begin{aligned}
& p c_{v}[\psi] \cap p c_{v}[\varphi]=\varnothing, \\
& \forall \psi-\varphi \geq 2 ;
\end{aligned}
$$

2) in addition, the adjacent elements permitted the existence of common movement, so if the two elements separated by one element have common movement, it was necessary to ensure that the same movement existed between the two elements and the intermediate elements. In addition, it needed to be met that there was no common movement between other elements: 
$\left\{\begin{array}{l}p c_{\mathrm{v}}[\psi] \cap p c_{\mathrm{v}}[\varphi] \neq \varnothing ; \\ p c_{\mathrm{v}}[\psi] \cap p c_{\mathrm{v}}\left[\frac{\psi+\varphi}{2}\right] \cap p c_{\mathrm{v}}[\varphi] \neq \varnothing,\end{array}\right.$

$\forall \psi-\varphi=2$,

additionally,

$$
p c_{v}[\psi] \cap p c_{v}[\varphi]=\varnothing, \forall \psi-\varphi \geq 3
$$

Thus, the array Char $p c_{v}\left[m_{\mathrm{v}}\right]$ was an FPC scheme, which must simultaneously satisfy Equations (14) and (15) or Equations (14), (16a) and (16b).

To help understand, the determination process of all FPC schemes was described in Figure 5.

All FPC schemes of the intersection can be determined according to this method, which can be expressed as:

$$
\begin{aligned}
& \mathbf{F P C}_{w}=\left\{\text { Char } f p c_{w}\left[m_{w}\right],\right. \\
& \left.w \in N^{+} \text {and } m_{w} \in N^{+}\right\} .
\end{aligned}
$$

Each array Char $f p c_{w}\left[m_{w}\right]$ represented an FPC scheme. Overall FPC schemes can be determined by this method.

\section{Signal timing optimization model for the PMC scheme}

A series of the FPC schemes for the signal intersections can be obtained according to the above method. Because these schemes were more extensive than the conventional PC scheme (OS, DS, CS and RBS), the corresponding optimal timing model should be built for FPC schemes.

\subsection{Objective function}

Signal timing for urban intersections involve many parameters, such as cycle length, effective red and green times of the phases, and all-red intervals. Then, after the signal scheme had been implemented, there were many evaluation indexes of signal performance, such as signal delay, and queue length (Hao et al. 2012). Currently, most signal timing optimization models were based on the evaluation indexes of signal performance and establish the optimization model by considering constraints.

According to the above research, the PMC and FPC schemes had been obtained. A complete signal control scheme for intersections included two parts, the PC scheme and the signal-timing scheme. The main objective of this section was to build an optimal timing model for different FPC schemes. For the control performance analysis of intersection, delay and capacity were the most common two measures indexes of effectiveness ( $\mathrm{Wu}, \mathrm{Gi}-$ uliani 2016). Therefore, this paper established the optimal timing model based on the two evaluation indexes.

Many models were used to calculated the average delay and traffic capacity (Akçelik, Rouphail 1993), and the calculation model in the Highway Capacity Manual (TRB 2010) was typically used. The model in the Highway Capacity Manual put forward the concept of lane group, which divided each intersection approach into several lane groups. According to this concept, when the approach of the intersection did not have the shared lane, every movement was a lane group. Otherwise, all movements in an approach were considered as one lane group. Therefore, the average delay of the movement $\sigma_{i j}$ (a lane group) in $\mathbf{F P C}_{w}$ was as follows:

$$
\begin{aligned}
& A D_{i j}^{w}=A D_{i j}^{w, 1}+A D_{i j}^{w, 2}+A D_{i j}^{w, 3} ; \\
& A D_{i j}^{w, 1}=\frac{0.5 \cdot C^{w} \cdot\left(1-\frac{G e_{i j}^{w}}{C^{w}}\right)^{2}}{1-\left(\min \left(1, \xi_{i j}^{w}\right) \cdot \frac{G e_{i j}^{w}}{C^{w}}\right)} ; \\
& A D_{i j}^{w, 2}=900 \cdot T_{a p} \times \\
& \left(\begin{array}{l}
\left(\xi_{i j}^{w}-1\right)+\sqrt{\left(\xi_{i j}^{w}-1\right)^{2}+\frac{8 \cdot r \cdot f \cdot \xi_{i j}^{w}}{C L G_{i j}^{w} \cdot T_{a p}}}
\end{array} .\right.
\end{aligned}
$$

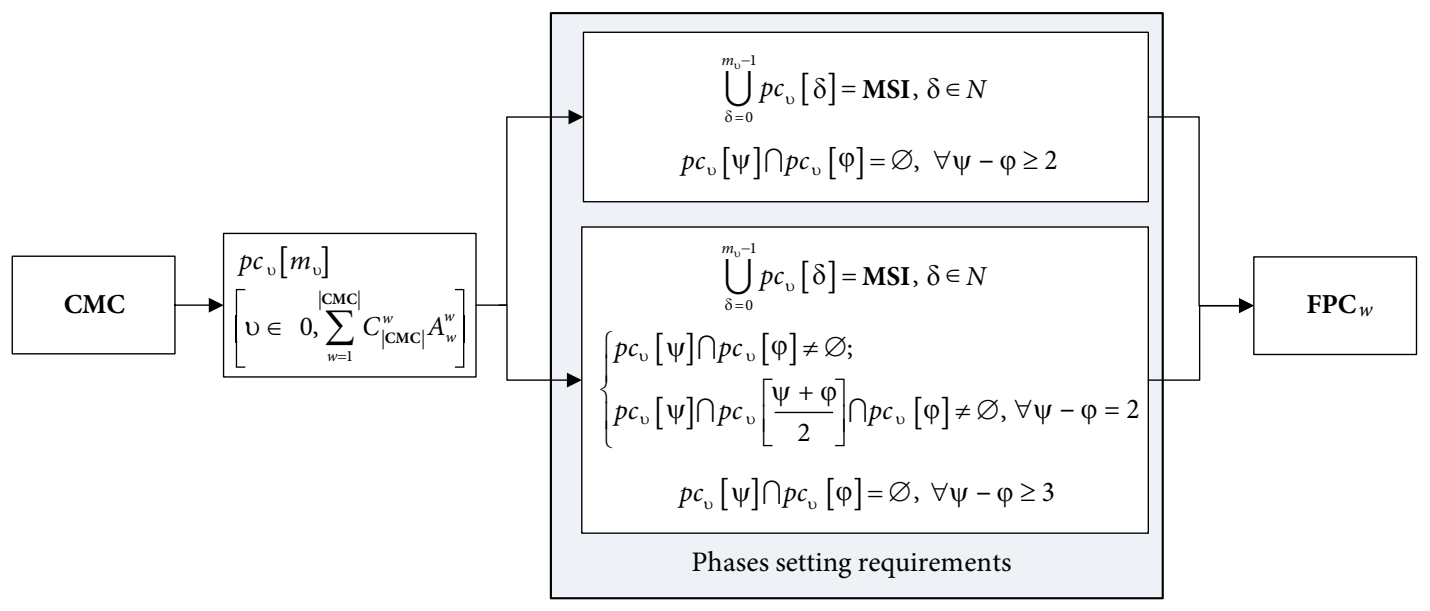

Figure 5. Determination procedure of the FPC scheme 
For the volume-to-capacity ratio:

$$
\xi_{i j}^{w}=\frac{Q_{i j} \cdot C^{w}}{N_{i j} \cdot S_{i j}^{\ln } \cdot G e_{i j}^{w}} .
$$

The calculation model of lane group capacity can be calculated as:

$$
C L G_{i j}^{w}=\frac{N_{i j} \cdot S_{i j}^{l n} \cdot G e_{i j}^{w}}{C^{w}},
$$

where: $Q_{i j}$ is the demand flow rate; $N_{i j}$ is the number of lanes in a lane group (movement $\sigma_{i j}$ ); $S_{i j}^{l n}$ is one lane saturation flow rate of the movement $\sigma_{i j}$.

After defining the average delay and capacity of the lane group, the average delay and capacity of every approach can also be calculated in Equations (23a) and (23b):

$$
\begin{gathered}
A D_{i}^{w}=\frac{\sum_{j=1}^{N_{i}} Q_{i j} \cdot A D_{i j}^{w}}{\sum_{j=1}^{N_{i}} Q_{i j}} ; \\
C L G_{i}^{w}=\sum_{j=1}^{N_{i}} C L G_{i j}^{w},
\end{gathered}
$$

where: $N_{i}$ is the number of movements in approach $i$.

However, when the approach of the intersection had a shared lane, all movements in an approach were one lane group. The average delay and capacity of the approach $i$ with shared lane can be calculated as following:

$$
\left\{\begin{array}{l}
A D_{i \mid e_{i 2}=1}^{w}=E q n_{1} \\
A D_{i \mid e_{i 2}=1}^{w, 1}=E q n_{2} \\
A D_{i \mid e_{i 2}=1}^{w, 2}=E q n_{3}
\end{array}\right.
$$

where:

$$
\begin{aligned}
& E q n_{1}=A D_{i \mid e_{i 2}=1}^{w, 1}+A D_{i \mid e_{i 2}=1}^{w, 2}+A D_{i \mid e_{i 2}=1}^{w, 3} ; \\
& E q n_{2}=\frac{0.5 \cdot C^{w} \cdot\left(1-\frac{G e_{i \mid e_{i 2}=1}^{w}}{C^{w}}\right)^{2}}{1-\left(\min \left(1, \xi_{i \mid e_{i 2}=1}^{w}\right) \cdot \frac{G e_{i \mid e_{i 2}=1}^{w}}{C^{w}}\right)} ; \\
& E q n_{3}=900 \cdot T_{a p} \times \\
& \left(\left(\xi_{i \mid e_{i 2}=1}^{w}-1\right)+\sqrt{\left(\xi_{i \mid e_{i 2}=1}^{w}-1\right)^{2}+\frac{8 \cdot r \cdot f \cdot \xi_{i \mid e_{i 2}=1}^{w}}{C L G_{i \mid e_{i 2}=1}^{w} \cdot T_{a p}}}\right)
\end{aligned}
$$

and

$$
\left\{\begin{array}{l}
\xi_{i \mid e_{i 2}=1}^{w}=\frac{Q_{i \mid e_{i 2}=1} \cdot C^{w}}{N_{i||_{i 2}=1} \cdot S_{i \mid e_{i 2}=1}^{\ln } \cdot G e_{i \mid e_{i 2}=1}^{w}} ; \\
C L G_{i \mid e_{i 2}=1}^{w}=\frac{N_{i \mid e_{i 2}=1} \cdot S_{i||_{i 2}=1}^{\ln } \cdot G e_{i \mid e_{i 2}=1}^{w}}{C^{w}} .
\end{array}\right.
$$

The meaning of the symbol in Equations (24) and (25) corresponded to the above, and $G e_{\left.i\right|_{i 2}=1}^{w}$ is the effective green time of the approach with shared lane $G e_{i \mid e_{i 2}=1}^{w}=$ $G e_{i j \mid e_{i 2}=1}^{w} \cdot Q_{i \mid e_{i 2}=1}$ is the traffic flow rate of the approach with shared lane. $N_{i||_{i 2}=1}$ is the number of movements in approach $i$ with shared lane. Therefore, the average delay and capacity of the approach $i$ with shared lane in $\mathbf{F P C}_{w}$ were as follows:

$$
\begin{aligned}
& A D_{i}^{w}=A D_{i \mid e_{i 2}=1}^{w} ; \\
& C L G_{i}^{w}=C L G_{i \mid e_{i 2}=1}^{w} .
\end{aligned}
$$

After defining the average delay and capacity of every approach, the average delay and capacity of the entire intersection can be calculated as:

$$
\begin{gathered}
A D^{w}=\frac{\sum_{i=1}^{I} Q_{i} \cdot A D_{i}^{w}}{\sum_{i=1}^{I} Q_{i}} ; \\
C L G^{w}=\sum_{i=1}^{I} C L G_{i}^{w},
\end{gathered}
$$

where: $I$ is the number of approaches in the intersection.

In generally, the average vehicle delay was expected to have a minimum value, while the traffic capacity was expected to have a maximum value. Therefore, Equation (28a) minimized the average vehicle delay, and Equation (28b) maximized the intersection capacity:

$$
\begin{gathered}
\min A D^{w}=\frac{\sum_{i=1}^{I} Q_{i} \cdot A D_{i}^{w}}{\sum_{i=1}^{I} Q_{i}} ; \\
\max C L G^{w}=\sum_{i=1}^{I} C L G_{i}^{w} .
\end{gathered}
$$

To solve the signal timing, the multi-objective optimization problem was typically transformed into a single objective optimization problem. Therefore, after calculating the capacity calculation model, the two-calculation model was summed. Finally, the objective function of the single objective optimal problem can be determined as shown in Equation (29):

$$
\begin{aligned}
& \min S O^{w}=A D^{w}+\frac{3600}{C L G^{w}}= \\
& \frac{\sum_{i=1}^{I} Q_{i} \cdot A D_{i}^{w}}{\sum_{i=1}^{I} Q_{i}}+\frac{3600}{\sum_{i=1}^{I} C L G_{i}^{w}} .
\end{aligned}
$$

\subsection{Constraints}

Obviously, the variables in the objective function were the effective green time of the movement $G e_{i j}^{w}$. To facilitate the optimization of the objective function, $G e_{i j}^{w}$ needed to 
be transformed with the effective green time of the corresponding phase $G e p_{i j}{ }_{i j} l_{i j}^{w, k}$.

According to Equation (17), the array $C h a r f p c_{w}\left[m_{w}\right]$ represented the $\mathbf{F P C}_{w}$ consisting of some CMCs. The phase number of the $\mathbf{F P C}$ was the element number $m_{w}$ in the array Char $f p c_{w}\left[m_{w}\right], f p c_{w}[x]$ was one element in the array Char $f p c_{w}\left[m_{w}\right], x=\left[0, m_{w}-1\right]$ and $x \in N^{+}$, and $f p c_{w}[x]$ represented the $(x+1)$ th phase in $\mathbf{F P C}$. $\eta_{i j}^{w}$ was the number of elements that contained movement $\sigma_{i j}$ in Char $f p c_{w}\left[m_{w}\right], \eta_{i j}^{w}=[1,3]$ and $\eta_{i j}^{w} \in N^{+} . x_{i j}^{w}$,k was the identifier of the element that contained movement $\sigma_{i j}$ in $C h a r f p c_{w}\left[m_{w}\right], \quad x_{i j}^{w, k}=\left[0, m_{w}-1\right]$ and $x_{i j}^{w, k} \in N^{+}$. $l_{i j}^{w, k}$ was the identifier of the phase that contained movement $\sigma_{i j}$ in $\mathbf{F P C}_{w}$. The identifier of the first element was 0 , so $l_{i j}^{w, k}=x_{i j}^{w, k}+1$. The parameter symbols of movement $\sigma_{i j}$ were shown in Table 2.

Then,

$$
G e_{i j}^{w}=\sum_{k=1}^{\eta_{i j}^{w}} G e p_{i j}^{w, l_{i j}^{w, k}}=\sum_{k=1}^{\eta_{i j}^{w}} G e p_{i j}^{w, x_{i j}^{w}}{ }^{w, k}+1
$$

The parameter relations of movement $\sigma_{i j}$ in $\mathbf{F P C}$ are were shown in Table 3.

Then,

$$
\begin{aligned}
& C^{w}=\sum_{u=1}^{m_{w}} T^{w, u} ; \\
& R e_{i j}^{w}=C^{w}-G e_{i j}^{w} .
\end{aligned}
$$

Considering the time that the driver can wait and the safety of traffic at the intersection, $C^{w}$ and $G_{i j}^{w}$ needed to

Table 2. Parameter symbols of movement $\sigma_{i j}$

\begin{tabular}{|l|l|l|c|}
\hline Number & \multicolumn{1}{|c|}{ Value } & \multicolumn{1}{|c|}{ Identifier } & \multicolumn{1}{c|}{ Size } \\
\hline$\eta_{i j}^{w}=1$ & $k=1$ & $x_{i j}^{w, 1}$ & - \\
\hline$\eta_{i j}^{w}=2$ & $k=1,2$ & $x_{i j}^{w, 1}, x_{i j}^{w, 2}$ & $x_{i j}^{w, 1}<x_{i j}^{w, 2}$ \\
\hline$\eta_{i j}^{w}=3$ & $k=1,2,3$ & $x_{i j}^{w, 1}, x_{i j}^{w, 2}, x_{i j}^{w, 3}$ & $x_{i j}^{w, 1}<x_{i j}^{w, 2}<x_{i j}^{w, 3}$ \\
\hline
\end{tabular}

obey the constraints in Equations (33) and (34):

$$
\begin{aligned}
& C_{\min } \leq C^{w} \leq C_{\max } \\
& G_{\min } \leq G_{i j}^{w} \leq G_{\max }, \forall \sigma_{i j} .
\end{aligned}
$$

The intersection signal phase was composed of different movements. Because a max-min constraint existed on the green time of movements, the constraints of the phase green time should be considered in detail. When the movement combinations of a phase were different, the green time needed to obey different constraint conditions. The array element $f p c_{w}[x]$ represented a signal phase, and there were two or three movements in it. Therefore, this paper analysed and discussed the constraint conditions of the phase effective green time under different cases to obey the movement constraints of the green time by considering the composition of different movements in $f p c_{w}[x]$. The specific analysis of each case was as follows.

Case 1. If there were three movements in $f p c_{w}[x]$, the three movements were in an approach, and one was the movement of the shared lane. Assumed that the approach was $i$, the three movements were $\sigma_{i 1}, \sigma_{i 2}$ and $\sigma_{i 3}$. The movements were also in the same phase, $l_{i 1}^{w, 1}=l_{i 2}^{w, 2}=l_{i 3}^{w, 3}$. $\sigma_{i 1}, \sigma_{i 2}$ and $\sigma_{i 3}$ were all non-lapping movements (the non-lapping movement occurred in only one phase), so $\eta_{i 1}^{w}=\eta_{i 2}^{w}=\eta_{i 3}^{w}=1$ and:

$$
\begin{aligned}
& G e_{i 1}^{w}=G e p_{i 1}^{w},_{i 1}^{w, 1}= \\
& G e_{i 2}^{w}=G e p_{i 2}^{w},_{i 2}^{w, 1}= \\
& G e_{i 3}^{w}=G e p_{i 3}^{w}{ }_{i 3}^{w, 1} .
\end{aligned}
$$

In Table 3, Gep $p_{i 1}^{w},_{i 1}^{w 1}=G p_{i 1}^{w}$, $_{i 1}^{w 1}+A-L, j=1,2,3$. Thus, according to Equations (35a) and (34), $\sigma_{i 1}, \sigma_{i 2}$ and $\sigma_{i 3}$

\begin{tabular}{|c|c|c|c|c|}
\hline Number & Phases & Effective green time of phase & Display green time & Phase time \\
\hline$\eta_{i j}^{w}=1$ & $l_{i j}^{w}, 1$ & $G e p_{i j}^{w, l_{i j}^{w, 1}}$ & $G p_{i j}^{w, l_{i j}^{w, 1}}=G e p_{i j}^{w, l_{i j}^{w, 1}}+L-A$ & $T_{i j}^{w, l_{i j}^{w, 1}}=G p_{i j}^{w, l_{i j}^{w, 1}}+A$ \\
\hline$\eta_{i j}^{w}=2$ & $\begin{array}{l}l_{i j}^{w, 1} \\
l_{i j}^{w, 2}\end{array}$ & $\begin{array}{l}G e p_{i j}^{w, l_{i j}^{w, 1}} \\
G e p_{i j}^{w, l_{i j}^{w, 2}}\end{array}$ & $\begin{array}{l}G p_{i j}^{w, l_{i j}^{w, 1}}=G e p_{i j}^{w, l_{i j}^{w, 1}}+L ; \\
G p_{i j}^{w, l_{i j}^{w, 2}}=G e p_{i j}^{w, l_{i j}^{w, 2}}-A\end{array}$ & $\begin{array}{l}T_{i j}^{w, l_{i j}^{w, 1}}=G p_{i j}^{w, l_{i j}^{w, 1}} ; \\
T_{i j}^{w, l_{i j}^{w, 2}}=G p_{i j}^{w, l_{i j}^{w, 2}}+A\end{array}$ \\
\hline$\eta_{i j}^{w}=3$ & $\begin{array}{l}l_{i j}^{w, 1} \\
l_{i j}^{w, 2} \\
l_{i j}^{w, 3}\end{array}$ & $\begin{array}{l}G e p_{i j}^{w, l_{i j}^{w, 1}} \\
G e p_{i j}^{w, l_{i j}^{w, 2}} \\
G e p_{i j}^{w, l_{i j}^{w, 3}}\end{array}$ & $\begin{array}{l}G p_{i j}^{w, l_{i j}^{w, 1}}=G e p_{i j}^{w, l_{i j}^{w, 1}}+L ; \\
G p_{i j}^{w, l_{i j}^{w, 2}}=G e p_{i j}^{w, l_{i j}^{w, 2}} ; \\
G p_{i j}^{w_{i j} l_{i j}^{w, 3}}=G e p_{i j}^{w, l_{i j}^{w, 3}}-A\end{array}$ & $\begin{array}{l}T_{i j}^{w, l_{i j}^{w, 1}}=G p_{i j}^{w, l_{i j}^{w, 1}} ; \\
T_{i j}^{w, l_{i j}^{w, 2}}=G p_{i j}^{w, l_{i j}^{w}, 2} ; \\
T_{i j}^{w, l_{i j}^{w, 3}}=G p_{i j}^{w, l_{i j}^{w, 3}}+A\end{array}$ \\
\hline
\end{tabular}
corresponded to the phase effective green time that should obey Equation (35b):

$$
\begin{aligned}
& G_{\min }+A-L \leq G e p_{i 1}^{w} l_{i 1}^{w 1}=G e p_{i 2}^{w}, l_{i 2}^{w, 1}= \\
& G e p_{i 3}^{w, l_{i 3}^{w, 1}} \leq G_{\max }+A-L .
\end{aligned}
$$

In addition, when 2 movements exited in $f p c_{w}[x]$, assumed that they were $\sigma_{i j}$ and $\sigma_{\mu \lambda}, \mu$ and $i, \lambda$ and $j$ had

Table 3. Parameter relations of movement $\sigma_{i j}$

Notes: $L$ is the lost time; $A$ is the yellow time. 
the same meaning. The two movements in one phase were different, so $\sigma_{i j} \neq \sigma_{\mu \lambda}$; however, the two movements in a phase corresponding to the phase time length were equal:

$$
T_{i j}^{w, l_{i j}^{w, k}}=T_{\mu \lambda}^{w, l_{\mu \lambda}^{w, k^{\prime}}}
$$

where: $k$ and $k^{\prime}$ were the same meaning in the above equation.

Moreover, $\sigma_{i j}$ and $\sigma_{\mu \lambda}$ may be the lapping movement, so the relationship between $G e p_{i j}^{w, l_{i j}^{w, k}}$ and $G e p_{\mu \lambda}^{w, l_{\mu \lambda}^{w, k^{\prime}}}$ needed to be determined in accordance with the following cases.

Case 2. The two movements $\left(\sigma_{i j}\right.$ and $\left.\sigma_{\mu \lambda}\right)$ in $f p c_{w}[x]$ were both non-lapping movements, so $\eta_{i j}^{w}=\eta_{\mu \lambda}^{w}=1$, $k=k^{\prime}=1$. Additionally:

$$
\left\{\begin{array}{l}
l_{i j}^{w, 1}=l_{\mu \lambda}^{w, 1} ; \\
G_{i j}^{w}=G p_{i j}^{w, l_{i j}^{w, 1}}=G_{\mu \lambda}^{w}=G p_{\mu \lambda}^{w, l_{\mu \lambda}^{w, 1}} ; \\
G e_{i j}^{w}=G e p_{i j}^{w, l_{i j}^{w, 1}}=G e_{\mu \lambda}^{w}=G e p_{\mu \lambda}^{w, l_{\mu \lambda}^{w, 1}} .
\end{array}\right.
$$

$G e p_{i j}^{w, l_{i j}^{w, 1}}=G p_{i j}^{w, l_{i j}^{w, 1}}+A-L$, and $G e p_{\mu \lambda}^{w, l_{\mu \lambda}^{w, 1}}=G p_{\mu \lambda}^{w, \lambda_{\mu \lambda}^{w, 1}}+$ $A-L$, according to Equations (37a) and (34):

$$
\begin{gathered}
G_{\min }+A-L \leq G e p_{i j}^{w, l_{i j}^{w, 1}}= \\
G e p_{\mu \lambda}^{w, l_{\mu \lambda}^{w, 1}} \leq G_{\max }+A-L .
\end{gathered}
$$

Case 3. One of two movements $\left(\sigma_{i j}\right.$ and $\left.\sigma_{\mu \lambda}\right)$ in $f p c_{w}[x]$ was the non-lapping movement; assumed that $\sigma_{\mu \lambda}$ was the lapping movement, $\eta_{i j}^{w} \neq 1$ and $\eta_{\mu \lambda}^{w}=1$, so $k^{\prime}=1$. Additionally:

$$
\left\{\begin{array}{l}
l_{i j}^{w, k}=l_{\mu \lambda}^{w, 1} ; \\
G_{\mu \lambda}^{w}=G p_{\mu \lambda}^{w, l_{\mu \lambda}^{w, 1}}=G p^{w, l_{\mu \lambda}^{w, 1}}=T_{i j}^{w, l_{i j}^{w, k}}-A, \quad \forall k ; \\
G e_{\mu \lambda}^{w}=G e p_{\mu \lambda}^{w, l_{\mu \lambda}^{w, 1}}=G e p_{i j}^{w, l_{i j}^{w, k}}, k=1 ; \\
G e_{\mu \lambda}^{w}=G e p_{\mu \lambda}^{w, l_{\mu \lambda}^{w, 1}}=G e p_{i j}^{w, l_{i j}^{w, k}}-L, k \neq 1 .
\end{array}\right.
$$

$G e p_{\mu \lambda}^{w, l_{\mu \lambda}^{w, 1}}=G p_{\mu \lambda}^{w, l_{\mu \lambda}^{w, 1}}+A-L$, according to Equations (38a) and (34):

$$
G_{\min }+A-L \leq G e p_{\mu \lambda}^{w, l_{\mu \lambda}^{w, 1}} \leq G_{\max }+A-L
$$

Case 4. The two movements $\left(\sigma_{i j}\right.$ and $\left.\sigma_{\mu \lambda}\right)$ in $f p c_{w}[x]$ were the lapping movements, $\eta_{i j}^{w} \geq 2$ and $\eta_{\mu \lambda}^{w} \geq 2$. The two movements in the same phase were different, so $k \neq k^{\prime}$ and:

$$
\left\{\begin{array}{l}
l_{i j}^{w, k}=l_{\mu \lambda}^{w, k^{\prime}} ; \\
G e p_{i j}^{w, l_{i j}^{w, k}}=G e p_{\mu \lambda}^{w, l_{\mu \lambda}^{w, k^{\prime}}}-L, k<k^{\prime} ; \\
G e p_{i j}^{w, l_{i j}^{w, k}}=G e p_{\mu \lambda}^{w, l_{\mu \lambda}^{w, k^{\prime}}}+L, k>k^{\prime} .
\end{array}\right.
$$

To ensure max-min green time constraints of the movements and the presence of phases, Equation (39b) should be given:

$$
0<T_{i j}^{w, l_{i j}^{w, k}}=T_{\mu \lambda}^{w, l_{\mu \lambda}^{w, k^{\prime}}} \leq G_{\max }+A-L
$$

The optimization model presented above can optimize signal timings, which was composed of the objective function in Equation (29) and the constraint conditions in Equations (30)-(39b). For a FPC scheme, the main variable of PM was $G e p_{i j}^{w, l_{i j}^{w, k}}$, and there were many related variables, such as $G e_{i j}^{w}, C^{w}, G p_{i j}^{w, l_{i j}^{w, k}}, T_{i j}^{w, l_{i j}^{w, k}}$ and constant variable, such as $A, L, \eta_{i j}^{w}, x_{i j}^{w, k}$ and $l_{i j}^{w, k}$. All related variables in the model can be represented by the main variables, so the optimization model can be transformed into an expression that contains only the main variable $G e p_{i j}^{w, l_{i j}^{w, k}}$. For the proposed optimization model, the objective function was non-linear, and all the constraints were linear. Therefore, the optimization model was a mixed-integer linear programming. In general, the mixed-integer-non-linear-programme can be linearized and converted into a mixed-integer linear programming, the specific linearization process can refer to Klanšek (2015). For mixed-integer linear programming, it can be solved by using the standard branch-and-bound technique (Zhao et al. 2015a). In the process of solving the model, we firstly determine the specific expression of the model for different FPC schemes by using programming code, and then use the toolbox of standard branch-and-bound technique to solve the optimization objective and all the variables, different FPC schemes correspond to different values of the objective. Finally, the control scheme of the corresponding minimum objective value will be selected as the adoption scheme to realize the optimal control of the intersection.

\section{Numerical analyses}

In this section, numerical experiments were discussed to support the theoretical results in the previous sections and to verify the feasibility of the proposed method.

\subsection{Performance evaluation}

For validating the PM, the intersection in Figure 6 was chosen as the study object, and the movement symbols were shown in figure.

According to the Section 3:

$$
\begin{aligned}
& \text { MSI }=\left\{\sigma_{11}, \sigma_{12}, \sigma_{13}, \sigma_{21}, \sigma_{23}, \sigma_{31}, \sigma_{33}, \sigma_{41}, \sigma_{43}\right\} ; \\
& e_{11}=e_{12}=e_{13}=e_{21}=e_{23}=e_{31}=e_{33}=e_{41}=e_{43}=1 ; \\
& e_{22}=e_{32}=e_{42}=0 .
\end{aligned}
$$

Movement compatibility can be determined, and the values of movement correlation parameters were shown in Table 4, according to table:

$$
\begin{aligned}
& \mathbf{C M C}=\left\{\left\{\sigma_{11}, \sigma_{12}, \sigma_{13}\right\},\left\{\sigma_{21}, \sigma_{23}\right\},\right. \\
& \left\{\sigma_{31}, \sigma_{33}\right\},\left\{\sigma_{41}, \sigma_{43}\right\},\left\{\sigma_{21}, \sigma_{41}\right\}, \\
& \left.\left\{\sigma_{23}, \sigma_{43}\right\},\left\{\sigma_{21}, \sigma_{33}\right\},\left\{\sigma_{31}, \sigma_{43}\right\}\right\},
\end{aligned}
$$

then, all FPC schemes can be determined, and there were 400 FPC schemes for the intersection in Figure 6 
Table 4. Values of movement correlation parameters

\begin{tabular}{|c|c|}
\hline Parameter & Value \\
\hline $\mathrm{CDM}_{\alpha}$ & $\begin{array}{l}\mathbf{C D M}_{1}=\left\{\sigma_{11}, \sigma_{12}, \sigma_{13}\right\} ; \\
\mathbf{C D M}_{2}=\left\{\sigma_{21}, \sigma_{23}\right\} ; \\
\mathbf{C D M}_{3}=\left\{\sigma_{31}, \sigma_{33}\right\} ; \\
\mathbf{C D M}_{4}=\left\{\sigma_{41}, \sigma_{43}\right\}\end{array}$ \\
\hline CDM & $\left\{\left\{\sigma_{11}, \sigma_{12}, \sigma_{13}\right\},\left\{\sigma_{21}, \sigma_{23}\right\},\left\{\sigma_{31}, \sigma_{33}\right\},\left\{\sigma_{41}, \sigma_{43}\right\}\right\}$ \\
\hline$C d m_{\alpha}$ & $C d m_{1}=C d m_{2}=C d m_{3}=C d m_{4}=1$ \\
\hline $\mathbf{C D M}_{\alpha \mid C d m_{\alpha}=1}$ & $\left\{\left\{\sigma_{11}, \sigma_{12}, \sigma_{13}\right\},\left\{\sigma_{21}, \sigma_{23}\right\},\left\{\sigma_{31}, \sigma_{33}\right\},\left\{\sigma_{41}, \sigma_{43}\right\}\right\}$ \\
\hline $\mathrm{COM}$ & $\begin{array}{l}\mathbf{C O M}_{1}=\left\{\sigma_{11}, \sigma_{31}\right\} ; \\
\mathbf{C O M}_{2}=\left\{\sigma_{13}, \sigma_{33}\right\} ; \\
\mathbf{C O M}_{3}=\left\{\sigma_{21}, \sigma_{41}\right\} ; \\
\mathbf{C O M}_{4}=\left\{\sigma_{23}, \sigma_{43}\right\}\end{array}$ \\
\hline $\mathrm{COM}$ & $\left\{\left\{\sigma_{11}, \sigma_{31}\right\},\left\{\sigma_{13}, \sigma_{33}\right\},\left\{\sigma_{21}, \sigma_{41}\right\},\left\{\sigma_{23}, \sigma_{43}\right\}\right\}$ \\
\hline $\operatorname{Com}_{\beta}$ & $\begin{array}{l}\operatorname{Com}_{1}=\operatorname{Com}_{2}=0 \\
\operatorname{Com}_{3}=\operatorname{Com}_{4}=1\end{array}$ \\
\hline $\mathrm{COM}_{\beta \mid \mathrm{Com}_{\beta}=1}$ & $\left\{\left\{\sigma_{21}, \sigma_{41}\right\},\left\{\sigma_{23}, \sigma_{43}\right\}\right\}$ \\
\hline $\mathbf{C C M}_{\gamma}$ & $\begin{array}{l}\mathbf{C C M}_{1}=\left\{\sigma_{11}, \sigma_{23}\right\} ; \\
\mathbf{C C M}_{2}=\left\{\sigma_{13}, \sigma_{41}\right\} ; \\
\mathbf{C C M}_{3}=\left\{\sigma_{21}, \sigma_{33}\right\} ; \\
\mathbf{C C M}_{4}=\left\{\sigma_{31}, \sigma_{43}\right\}\end{array}$ \\
\hline CCM & $\left\{\left\{\sigma_{11}, \sigma_{23}\right\},\left\{\sigma_{13}, \sigma_{41}\right\},\left\{\sigma_{21}, \sigma_{33}\right\},\left\{\sigma_{31}, \sigma_{43}\right\}\right\}$ \\
\hline $\mathrm{Ccm}_{\gamma}$ & $\begin{array}{l}\mathrm{Ccm}_{1}=\mathrm{Ccm}_{2}=0 \\
\mathrm{Ccm}_{3}=\mathrm{Ccm}_{4}=1\end{array}$ \\
\hline $\mathbf{C C M}_{\gamma \mid \mathrm{Ccm}_{\gamma}=1}$ & $\left\{\left\{\sigma_{21}, \sigma_{33}\right\},\left\{\sigma_{31}, \sigma_{43}\right\}\right\}$ \\
\hline
\end{tabular}

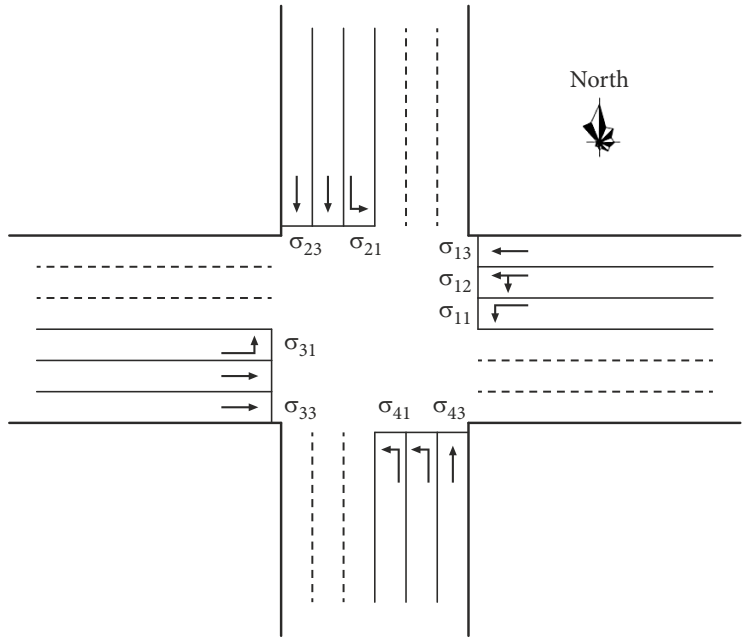

Figure 6. Numerical intersection

by programming calculations, include: 48 the four-phase schemes, 264 the five-phase schemes, and 88 the six-phase schemes, the partial FPC schemes were shown in Table 5.
Table 5. Partial FPC schemes

\begin{tabular}{|c|c|c|c|}
\hline$w$ & $m_{w}$ & FPC scheme & Phase \\
\hline 1 & 4 & $\left\{\sigma_{11}, \sigma_{12}, \sigma_{13}\right\},\left\{\sigma_{21}, \sigma_{23}\right\},\left\{\sigma_{31}, \sigma_{33}\right\},\left\{\sigma_{41}, \sigma_{43}\right\}$ & \multirow{7}{*}{4} \\
\hline 2 & 4 & $\left\{\sigma_{11}, \sigma_{12}, \sigma_{13}\right\},\left\{\sigma_{21}, \sigma_{23}\right\},\left\{\sigma_{41}, \sigma_{43}\right\},\left\{\sigma_{31}, \sigma_{33}\right\}$ & \\
\hline 3 & 4 & $\left\{\sigma_{11}, \sigma_{12}, \sigma_{13}\right\},\left\{\sigma_{31}, \sigma_{33}\right\},\left\{\sigma_{21}, \sigma_{23}\right\},\left\{\sigma_{41}, \sigma_{43}\right\}$ & \\
\hline 4 & 4 & $\left\{\sigma_{11}, \sigma_{12}, \sigma_{13}\right\},\left\{\sigma_{31}, \sigma_{33}\right\},\left\{\sigma_{41}, \sigma_{43}\right\},\left\{\sigma_{21}, \sigma_{23}\right\}$ & \\
\hline 5 & 4 & $\left\{\sigma_{11}, \sigma_{12}, \sigma_{13}\right\},\left\{\sigma_{31}, \sigma_{33}\right\},\left\{\sigma_{21}, \sigma_{41}\right\},\left\{\sigma_{23}, \sigma_{43}\right\}$ & \\
\hline 6 & 4 & $\left\{\sigma_{11}, \sigma_{12}, \sigma_{13}\right\},\left\{\sigma_{31}, \sigma_{33}\right\},\left\{\sigma_{23}, \sigma_{43}\right\},\left\{\sigma_{21}, \sigma_{41}\right\}$ & \\
\hline$\vdots$ & $\vdots$ & $\vdots$ & \\
\hline 49 & 5 & $\begin{array}{l}\left\{\sigma_{11}, \sigma_{12}, \sigma_{13}\right\},\left\{\sigma_{21}, \sigma_{23}\right\},\left\{\sigma_{31}, \sigma_{33}\right\} \\
\left\{\sigma_{31}, \sigma_{43}\right\},\left\{\sigma_{41}, \sigma_{43}\right\}\end{array}$ & \multirow{7}{*}{5} \\
\hline 50 & 5 & $\begin{array}{l}\left\{\sigma_{11}, \sigma_{12}, \sigma_{13}\right\},\left\{\sigma_{21}, \sigma_{23}\right\},\left\{\sigma_{41}, \sigma_{43}\right\} \\
\left\{\sigma_{31}, \sigma_{43}\right\},\left\{\sigma_{31}, \sigma_{33}\right\}\end{array}$ & \\
\hline 51 & 5 & $\begin{array}{l}\left\{\sigma_{11}, \sigma_{12}, \sigma_{13}\right\},\left\{\sigma_{21}, \sigma_{23}\right\},\left\{\sigma_{21}, \sigma_{41}\right\} \\
\left\{\sigma_{31}, \sigma_{33}\right\},\left\{\sigma_{31}, \sigma_{43}\right\}\end{array}$ & \\
\hline 52 & 5 & $\begin{array}{l}\left\{\sigma_{11}, \sigma_{12}, \sigma_{13}\right\},\left\{\sigma_{21}, \sigma_{23}\right\},\left\{\sigma_{21}, \sigma_{41}\right\}, \\
\left\{\sigma_{41}, \sigma_{43}\right\},\left\{\sigma_{31}, \sigma_{33}\right\}\end{array}$ & \\
\hline 53 & 5 & $\begin{array}{l}\left\{\sigma_{11}, \sigma_{12}, \sigma_{13}\right\},\left\{\sigma_{21}, \sigma_{23}\right\},\left\{\sigma_{21}, \sigma_{41}\right\} \\
\left\{\sigma_{21}, \sigma_{33}\right\},\left\{\sigma_{31}, \sigma_{43}\right\}\end{array}$ & \\
\hline 54 & 5 & $\begin{array}{l}\left\{\sigma_{11}, \sigma_{12}, \sigma_{13}\right\},\left\{\sigma_{21}, \sigma_{23}\right\},\left\{\sigma_{21}, \sigma_{41}\right\} \\
\left\{\sigma_{31}, \sigma_{43}\right\},\left\{\sigma_{31}, \sigma_{33}\right\}\end{array}$ & \\
\hline$\vdots$ & $\vdots$ & $\vdots$ & \\
\hline 313 & 6 & $\begin{array}{l}\left\{\sigma_{11}, \sigma_{12}, \sigma_{13}\right\},\left\{\sigma_{21}, \sigma_{23}\right\},\left\{\sigma_{21}, \sigma_{41}\right\} \\
\left\{\sigma_{41}, \sigma_{43}\right\},\left\{\sigma_{31}, \sigma_{43}\right\},\left\{\sigma_{31}, \sigma_{33}\right\}\end{array}$ & \multirow{10}{*}{6} \\
\hline 314 & 6 & $\begin{array}{l}\left\{\sigma_{11}, \sigma_{12}, \sigma_{13}\right\},\left\{\sigma_{21}, \sigma_{23}\right\},\left\{\sigma_{21}, \sigma_{41}\right\} \\
\left\{\sigma_{21}, \sigma_{33}\right\},\left\{\sigma_{31}, \sigma_{33}\right\},\left\{\sigma_{31}, \sigma_{43}\right\}\end{array}$ & \\
\hline 315 & 6 & $\begin{array}{l}\left\{\sigma_{11}, \sigma_{12}, \sigma_{13}\right\},\left\{\sigma_{21}, \sigma_{23}\right\},\left\{\sigma_{23}, \sigma_{43}\right\} \\
\left\{\sigma_{41}, \sigma_{43}\right\},\left\{\sigma_{31}, \sigma_{43}\right\},\left\{\sigma_{31}, \sigma_{33}\right\}\end{array}$ & \\
\hline 316 & 6 & $\begin{array}{l}\left\{\sigma_{11}, \sigma_{12}, \sigma_{13}\right\},\left\{\sigma_{21}, \sigma_{23}\right\},\left\{\sigma_{21}, \sigma_{33}\right\}, \\
\left\{\sigma_{31}, \sigma_{33}\right\},\left\{\sigma_{31}, \sigma_{43}\right\},\left\{\sigma_{41}, \sigma_{43}\right\}\end{array}$ & \\
\hline 317 & 6 & $\begin{array}{l}\left\{\sigma_{11}, \sigma_{12}, \sigma_{13}\right\},\left\{\sigma_{21}, \sigma_{23}\right\},\left\{\sigma_{21}, \sigma_{33}\right\} \\
\left\{\sigma_{21}, \sigma_{41}\right\},\left\{\sigma_{41}, \sigma_{43}\right\},\left\{\sigma_{31}, \sigma_{43}\right\}\end{array}$ & \\
\hline 318 & 6 & $\begin{array}{l}\left\{\sigma_{11}, \sigma_{12}, \sigma_{13}\right\},\left\{\sigma_{31}, \sigma_{33}\right\},\left\{\sigma_{21}, \sigma_{33}\right\} \\
\left\{\sigma_{21}, \sigma_{23}\right\},\left\{\sigma_{21}, \sigma_{41}\right\},\left\{\sigma_{41}, \sigma_{43}\right\}\end{array}$ & \\
\hline$\vdots$ & $\vdots$ & $\begin{array}{r}\vdots \\
\end{array}$ & \\
\hline 389 & 6 & $\begin{array}{l}\left\{\sigma_{31}, \sigma_{43}\right\},\left\{\sigma_{23}, \sigma_{43}\right\},\left\{\sigma_{21}, \sigma_{23}\right\}, \\
\left\{\sigma_{21}, \sigma_{41}\right\},\left\{\sigma_{21}, \sigma_{33}\right\},\left\{\sigma_{11}, \sigma_{12}, \sigma_{13}\right\}\end{array}$ & \\
\hline 399 & 6 & $\begin{array}{l}\left\{\sigma_{31}, \sigma_{43}\right\},\left\{\sigma_{23}, \sigma_{43}\right\},\left\{\sigma_{21}, \sigma_{23}\right\}, \\
\left\{\sigma_{21}, \sigma_{33}\right\},\left\{\sigma_{21}, \sigma_{41}\right\},\left\{\sigma_{11}, \sigma_{12}, \sigma_{13}\right\}\end{array}$ & \\
\hline 400 & 6 & $\begin{array}{l}\left\{\sigma_{31}, \sigma_{43}\right\},\left\{\sigma_{23}, \sigma_{43}\right\},\left\{\sigma_{41}, \sigma_{43}\right\}, \\
\left\{\sigma_{21}, \sigma_{41}\right\},\left\{\sigma_{21}, \sigma_{33}\right\},\left\{\sigma_{11}, \sigma_{12}, \sigma_{13}\right\}\end{array}$ & \\
\hline
\end{tabular}


For a signalized intersection, the vehicle arrival of movements has a certain randomness (Cai et al. 2013). The collection of the actual data of vehicles is difficult since vehicles do not have the communication device. But with the continuous popularization and perfection of the technology of automated vehicles, data exchange can be conveniently carried out between different vehicles and infrastructures. Therefore, under automated vehicles environment, the vehicles could use mechatronics technology to drive autonomously, and share the different types of traffic parameters include individual vehicle manoeuvres, location, speed, trajectory, size and so on, with other vehicles and infrastructure in real time (Lee, Park 2012). In order to verify the effectiveness of the proposed md for different traffic conditions, three traffic demand scenarios were considered. assumed that the specific arrival of vehicles under different traffic demand scenarios listed in Table 6.

The proposed method and the signal-timing model were used to solve the corresponding optimal signal control scheme and the values of other parameters were listed as follows: minimum green time $G_{\min }=7 \mathrm{~s}$, maximum green time $G_{\max }=60 \mathrm{~s}$, common cycle length is between $C_{\min }=48 \mathrm{~s}$ and $C_{\max }=150 \mathrm{~s}$, saturation flow rate $S_{i j}=1800 \mathrm{veh} / \mathrm{h} /$ lane and yellow time $A=4 \mathrm{~s}$ (Liu, Chang 2011; Zhang, Wang 2011).

The optimal signal-timing model can be determined with the traffic volume and relevant parameter input. Every FPC scheme was different, so the different FPC schemes had different models. The corresponding optimal objective value and timing scheme can be obtained by solving the model, and the optimization results of the proposal method were shown in Figure 7. Comparing the objective values of all FPC schemes under different traffic demand scenarios, the control scheme corresponding to the minimum objective value was chosen as the optimal control scheme. The corresponding $w$ values were shown in the right half of Figure 7, and the optimization results of signal timings were shown in Table 7.

Because one approach of the intersection had a shared lane movement, according to the style of conventional PC schemes, the conventional intersection control schemes included only DS, CS and RBS (Table 8) under the left turn protection phase.

The optimal objective value and signal timing scheme can be obtained by optimizing the specific PC scheme, and the vehicle average delay and capacity under the different intersection control schemes can also be obtained by putting the signal phase timing parameters into the average delay and capacity models. The optimal target value, average delay and traffic capacity of different demand were different. The specific optimization results of objective values between PM corresponding to control schemes and the conventional schemes were shown in Table 9 and Figure 8. The numerical analyses showed that the overall objective values were improved, the traffic capacity was increased, and the average vehicle delay was further reduced than the conventional PC schemes. Therefore, the proposed method had a better control effectiveness.

\subsection{Case verification}

In order to further verify the feasibility and effectiveness of the proposed method, the above calculation results should be tested in VISSIM simulation software (https://www.ptvgroup.com/en/solutions/products/ptv-vissim). This section mainly simulated the PM schemes and the conventional PC schemes (DS, CS, RBS) and compares the simulation results. The optimal PM scheme and conventional PC schemes were put into the simulation software; the evaluation indexes (e.g., average delay, throughput, average queue length, and average number of stops) can be obtained by setting the detector in the simulation software. The simulation verification results were shown in Figure 9.

The corresponding evaluation index values of each control scheme in different traffic demand scenarios were obtained by simulation. The evaluation index value of the conventional signal control scheme was compared with the evaluation index value of the PM schemes individually. The analysis results showed that the evaluation index value of DS and RBS was less than that of the PM scheme when the traffic movement volumes were in the low-volume scenario and the control effectiveness was equivalent. For the medium- and high-volume scenarios, the evaluation index values of the PM scheme were improved compared with other schemes, especially for CS scheme, the degree of improvement was more remarkable. The analysis demonstrated that the parameter values of simulation results (Figure 9) matched with the calculation results of PM (Figure 8), which showed that the proposed method was effective and had practical significance to optimize the intersection control scheme by considering PMC.

Table 6. Traffic volume of the movements [veh/h]

\begin{tabular}{|c|c|c|c|c|c|c|c|c|}
\hline \multirow{2}{*}{$\begin{array}{l}\text { Demand } \\
\text { scenario }\end{array}$} & \multicolumn{2}{|c|}{$i=1$} & \multicolumn{2}{|c|}{$i=2$} & \multicolumn{2}{|c|}{$i=3$} & \multicolumn{2}{|c|}{$i=4$} \\
\hline & left & through & left & through & left & through & left & through \\
\hline \multirow[t]{2}{*}{ Low } & \multicolumn{2}{|c|}{750} & \multicolumn{2}{|c|}{600} & \multicolumn{2}{|c|}{950} & \multicolumn{2}{|c|}{1000} \\
\hline & 350 & 400 & 200 & 400 & 250 & 700 & 700 & 300 \\
\hline \multirow[t]{2}{*}{ Medium } & \multicolumn{2}{|c|}{1050} & \multicolumn{2}{|c|}{900} & \multicolumn{2}{|c|}{1250} & \multicolumn{2}{|c|}{1300} \\
\hline & 500 & 550 & 350 & 550 & 450 & 800 & 750 & 550 \\
\hline \multirow[t]{2}{*}{ High } & \multicolumn{2}{|c|}{1350} & \multicolumn{2}{|c|}{1200} & \multicolumn{2}{|c|}{1550} & \multicolumn{2}{|c|}{1600} \\
\hline & 650 & 700 & 450 & 750 & 550 & 1000 & 950 & 650 \\
\hline
\end{tabular}


Table 7. Optimization results of signal timings

\begin{tabular}{|c|c|c|c|c|c|c|c|c|c|c|c|c|c|c|}
\hline \multirow{3}{*}{$\begin{array}{l}\text { Demand } \\
\text { scenario }\end{array}$} & \multirow{3}{*}{$w$} & \multirow{3}{*}{ Optimization phases structure } & \multicolumn{10}{|c|}{ Optimization phase timings } & \multirow{3}{*}{$\begin{array}{c}\text { Cycle } \\
\text { length } \\
{[\mathrm{s}]}\end{array}$} & \multirow{3}{*}{$\begin{array}{c}S O^{w} \\
{[\mathrm{~s} / \mathrm{veh}]}\end{array}$} \\
\hline & & & \multirow{2}{*}{$\begin{array}{c}\text { Legs } \\
\text { Movements } \\
\end{array}$} & \multicolumn{3}{|c|}{1} & \multicolumn{2}{|c|}{2} & \multicolumn{2}{|c|}{3} & \multicolumn{2}{|c|}{4} & & \\
\hline & & & & $\sigma_{11}$ & $\sigma_{12}$ & $\sigma_{13}$ & $\sigma_{21}$ & $\sigma_{23}$ & $\sigma_{31}$ & $\sigma_{33}$ & $\sigma_{41}$ & $\sigma_{43}$ & & \\
\hline \multirow{12}{*}{ Low } & \multirow{3}{*}{321} & \multirow{3}{*}{$\begin{array}{l}\left\{\sigma_{11}, \sigma_{12}, \sigma_{13}\right\},\left\{\sigma_{31}, \sigma_{33}\right\},\left\{\sigma_{21}, \sigma_{33}\right\}, \\
\left\{\sigma_{21}, \sigma_{41}\right\},\left\{\sigma_{41}, \sigma_{43}\right\},\left\{\sigma_{23}, \sigma_{43}\right\}\end{array}$} & start of green $[\mathrm{s}]$ & 0 & 0 & 0 & 32 & 53 & 15 & 15 & 34 & 47 & \multirow{3}{*}{66} & \multirow{12}{*}{36.32680} \\
\hline & & & $\begin{array}{l}\text { duration of } \\
\text { green }[\mathrm{s}]\end{array}$ & 11 & 11 & 11 & 11 & 9 & 13 & 15 & 15 & 15 & & \\
\hline & & & end of green $[\mathrm{s}]$ & 11 & 11 & 11 & 43 & 62 & 28 & 30 & 49 & 62 & & \\
\hline & \multirow{3}{*}{337} & \multirow{3}{*}{$\begin{array}{l}\left\{\sigma_{11}, \sigma_{12}, \sigma_{13}\right\},\left\{\sigma_{23}, \sigma_{43}\right\},\left\{\sigma_{41}, \sigma_{43}\right\}, \\
\left\{\sigma_{21}, \sigma_{41}\right\},\left\{\sigma_{21}, \sigma_{33}\right\},\left\{\sigma_{31}, \sigma_{33}\right\}\end{array}$} & start of green $[\mathrm{s}]$ & 0 & 0 & 0 & 34 & 15 & 49 & 47 & 28 & 15 & \multirow{3}{*}{66} & \\
\hline & & & \begin{tabular}{|l|l} 
duration of \\
green $[\mathrm{s}]$
\end{tabular} & 11 & 11 & 11 & 11 & 9 & 13 & 15 & 15 & 15 & & \\
\hline & & & end of green [s] & 11 & 11 & 11 & 45 & 24 & 62 & 62 & 43 & 30 & & \\
\hline & \multirow{3}{*}{365} & \multirow{3}{*}{$\begin{array}{l}\left\{\sigma_{31}, \sigma_{33}\right\},\left\{\sigma_{21}, \sigma_{33}\right\},\left\{\sigma_{21}, \sigma_{41}\right\}, \\
\left\{\sigma_{41}, \sigma_{43}\right\},\left\{\sigma_{23}, \sigma_{43}\right\},\left\{\sigma_{11}, \sigma_{12}, \sigma_{13}\right\}\end{array}$} & start of green $[\mathrm{s}]$ & 51 & 51 & 51 & 17 & 38 & 0 & 0 & 19 & 32 & \multirow{3}{*}{66} & \\
\hline & & & $\begin{array}{l}\text { duration } \\
\text { of green }[\mathrm{s}]\end{array}$ & 11 & 11 & 11 & 11 & 9 & 13 & 15 & 15 & 15 & & \\
\hline & & & end of green [s] & 62 & 62 & 62 & 28 & & & & 34 & 47 & & \\
\hline & & & start of green [s] & 51 & 51 & 51 & 19 & 0 & 34 & 32 & 13 & 0 & & \\
\hline & 381 & $\begin{array}{l}\left\{\sigma_{23}, \sigma_{43}\right\},\left\{\sigma_{41}, \sigma_{43}\right\},\left\{\sigma_{21}, \sigma_{41}\right\}, \\
\left\{\sigma_{21}, \sigma_{33}\right\},\left\{\sigma_{31}, \sigma_{33}\right\},\left\{\sigma_{11}, \sigma_{12}, \sigma_{13}\right\}\end{array}$ & \begin{tabular}{|l|} 
duration \\
of green $[\mathrm{s}]$
\end{tabular} & 11 & 11 & 11 & 11 & 9 & 13 & 15 & 15 & 15 & 66 & \\
\hline & & & end of green [s] & 62 & 62 & 62 & 30 & 9 & 47 & 47 & 28 & 15 & & \\
\hline & & & start of green [s] & 0 & 0 & 0 & 26 & 26 & 52 & 48 & 84 & 78 & & \\
\hline & 316 & $\begin{array}{l}\left\{\sigma_{11}, \sigma_{12}, \sigma_{13}\right\},\left\{\sigma_{21}, \sigma_{23}\right\},\left\{\sigma_{21}, \sigma_{33}\right\} \\
\left\{\sigma_{31}, \sigma_{33}\right\},\left\{\sigma_{31}, \sigma_{43}\right\},\left\{\sigma_{41}, \sigma_{43}\right\}\end{array}$ & \begin{tabular}{|l|} 
duration \\
of green $[\mathrm{s}]$
\end{tabular} & 22 & 22 & 22 & 22 & 18 & 28 & 26 & 25 & 31 & 113 & \\
\hline & & & end of green [s] & 22 & 22 & 22 & 48 & 44 & 80 & 74 & 109 & 109 & & \\
\hline & & & start of green [s] & 0 & 0 & 0 & 87 & 91 & 55 & 61 & 26 & 26 & & \\
\hline & 329 & $\begin{array}{l}\left\{\sigma_{11}, \sigma_{12}, \sigma_{13}\right\},\left\{\sigma_{41}, \sigma_{43}\right\},\left\{\sigma_{31}, \sigma_{43}\right\}, \\
\left\{\sigma_{31}, \sigma_{33}\right\},\left\{\sigma_{21}, \sigma_{33}\right\},\left\{\sigma_{21}, \sigma_{23}\right\}\end{array}$ & \begin{tabular}{|l|}
$\begin{array}{l}\text { duration } \\
\text { of green }[\mathrm{s}]\end{array}$ \\
\end{tabular} & 22 & 22 & 22 & 22 & 18 & 28 & 26 & 25 & 31 & 113 & \\
\hline Medium & & & end of green [s] & 22 & 22 & 22 & 109 & 109 & 83 & 87 & 51 & 57 & & \\
\hline siedium & & & start of green $[\mathrm{s}]$ & 87 & 87 & 87 & 0 & 0 & 26 & 22 & 58 & 52 & & 19.29097 \\
\hline & 360 & $\begin{array}{l}\left\{\sigma_{21}, \sigma_{23}\right\},\left\{\sigma_{21}, \sigma_{33}\right\},\left\{\sigma_{31}, \sigma_{33}\right\}, \\
\left\{\sigma_{31}, \sigma_{43}\right\},\left\{\sigma_{41}, \sigma_{43}\right\},\left\{\sigma_{11}, \sigma_{12}, \sigma_{13}\right\}\end{array}$ & \begin{tabular}{|l|} 
duration \\
of green $[\mathrm{s}]$
\end{tabular} & 22 & 22 & 22 & 22 & 18 & 28 & 26 & 25 & 31 & 113 & \\
\hline & & & end of green [s] & 109 & 109 & 109 & 22 & 18 & 54 & 48 & 83 & 83 & & \\
\hline & & & start of green $[\mathrm{s}]$ & 87 & 87 & 87 & 61 & 65 & 29 & 35 & 0 & 0 & & \\
\hline & 373 & $\begin{array}{l}\left\{\sigma_{41}, \sigma_{43}\right\},\left\{\sigma_{31}, \sigma_{43}\right\},\left\{\sigma_{31}, \sigma_{33}\right\}, \\
\left\{\sigma_{21}, \sigma_{33}\right\},\left\{\sigma_{21}, \sigma_{23}\right\},\left\{\sigma_{11}, \sigma_{12}, \sigma_{13}\right\}\end{array}$ & \begin{tabular}{|l|} 
duration \\
of green $[\mathrm{s}]$
\end{tabular} & 22 & 22 & 22 & 22 & 18 & 28 & 26 & 25 & 31 & 113 & \\
\hline & & & end of green [s] & 109 & 109 & 109 & 83 & 83 & 57 & 61 & 25 & 31 & & \\
\hline & & & start of green $[\mathrm{s}]$ & 0 & 0 & 0 & 74 & 78 & 36 & 36 & 110 & 106 & & \\
\hline & 319 & $\begin{array}{l}\left\{\sigma_{11}, \sigma_{12}, \sigma_{13}\right\},\left\{\sigma_{31}, \sigma_{33}\right\},\left\{\sigma_{21}, \sigma_{33}\right\} \\
\left\{\sigma_{21}, \sigma_{23}\right\},\left\{\sigma_{23}, \sigma_{43}\right\},\left\{\sigma_{41}, \sigma_{43}\right\}\end{array}$ & $\begin{array}{l}\text { duration } \\
\text { of green [s] }\end{array}$ & 32 & 32 & 32 & 28 & 28 & 34 & 38 & 36 & 40 & 150 & \\
\hline & & & end of green [s] & 32 & 32 & 32 & 102 & 106 & 70 & 74 & 146 & 146 & & \\
\hline & & & start of green [s] & 0 & 0 & 0 & 80 & 76 & 112 & 108 & 36 & 36 & & \\
\hline & 327 & $\begin{array}{l}\left\{\sigma_{11}, \sigma_{12}, \sigma_{13}\right\},\left\{\sigma_{41}, \sigma_{43}\right\},\left\{\sigma_{23}, \sigma_{43}\right\}, \\
\left\{\sigma_{21}, \sigma_{23}\right\},\left\{\sigma_{21}, \sigma_{33}\right\},\left\{\sigma_{31}, \sigma_{33}\right\}\end{array}$ & \begin{tabular}{|l|} 
duration \\
of green $[\mathrm{s}]$
\end{tabular} & 32 & 32 & 32 & 28 & 28 & 34 & 38 & 36 & 40 & 150 & \\
\hline $\mathrm{High}$ & & & end of green [s] & 32 & 32 & 32 & 108 & 104 & 146 & 146 & 72 & 76 & & 158.50660 \\
\hline & & & start of green $[\mathrm{s}]$ & 114 & 114 & 114 & 38 & 42 & 0 & 0 & 74 & 70 & & \\
\hline & 363 & $\begin{array}{l}\left\{\sigma_{31}, \sigma_{33}\right\},\left\{\sigma_{21}, \sigma_{33}\right\},\left\{\sigma_{21}, \sigma_{23}\right\}, \\
\left\{\sigma_{23}, \sigma_{43}\right\},\left\{\sigma_{41}, \sigma_{43}\right\},\left\{\sigma_{11}, \sigma_{12}, \sigma_{13}\right\}\end{array}$ & $\begin{array}{l}\text { duration } \\
\text { of green }[\mathrm{s}] \\
\end{array}$ & 32 & 32 & 32 & 28 & 28 & 34 & 38 & 36 & 40 & 150 & \\
\hline & & & end of green [s] & 146 & 146 & 146 & 66 & 70 & 34 & 38 & 110 & 110 & & \\
\hline & & & start of green [s] & 114 & 114 & 114 & 44 & 40 & 76 & 72 & 0 & 0 & & \\
\hline & 371 & $\begin{array}{l}\left\{\sigma_{41}, \sigma_{43}\right\},\left\{\sigma_{23}, \sigma_{43}\right\},\left\{\sigma_{21}, \sigma_{23}\right\}, \\
\left\{\sigma_{21}, \sigma_{33}\right\},\left\{\sigma_{31}, \sigma_{33}\right\},\left\{\sigma_{11}, \sigma_{12}, \sigma_{13}\right\}\end{array}$ & \begin{tabular}{|l|}
$\begin{array}{l}\text { duration } \\
\text { of green }[\mathrm{s}]\end{array}$ \\
\end{tabular} & 32 & 32 & 32 & 28 & 28 & 34 & 38 & 36 & 40 & 150 & \\
\hline & & & end of green [s] & 146 & 146 & 146 & 72 & 68 & 110 & 110 & 36 & 40 & & \\
\hline
\end{tabular}



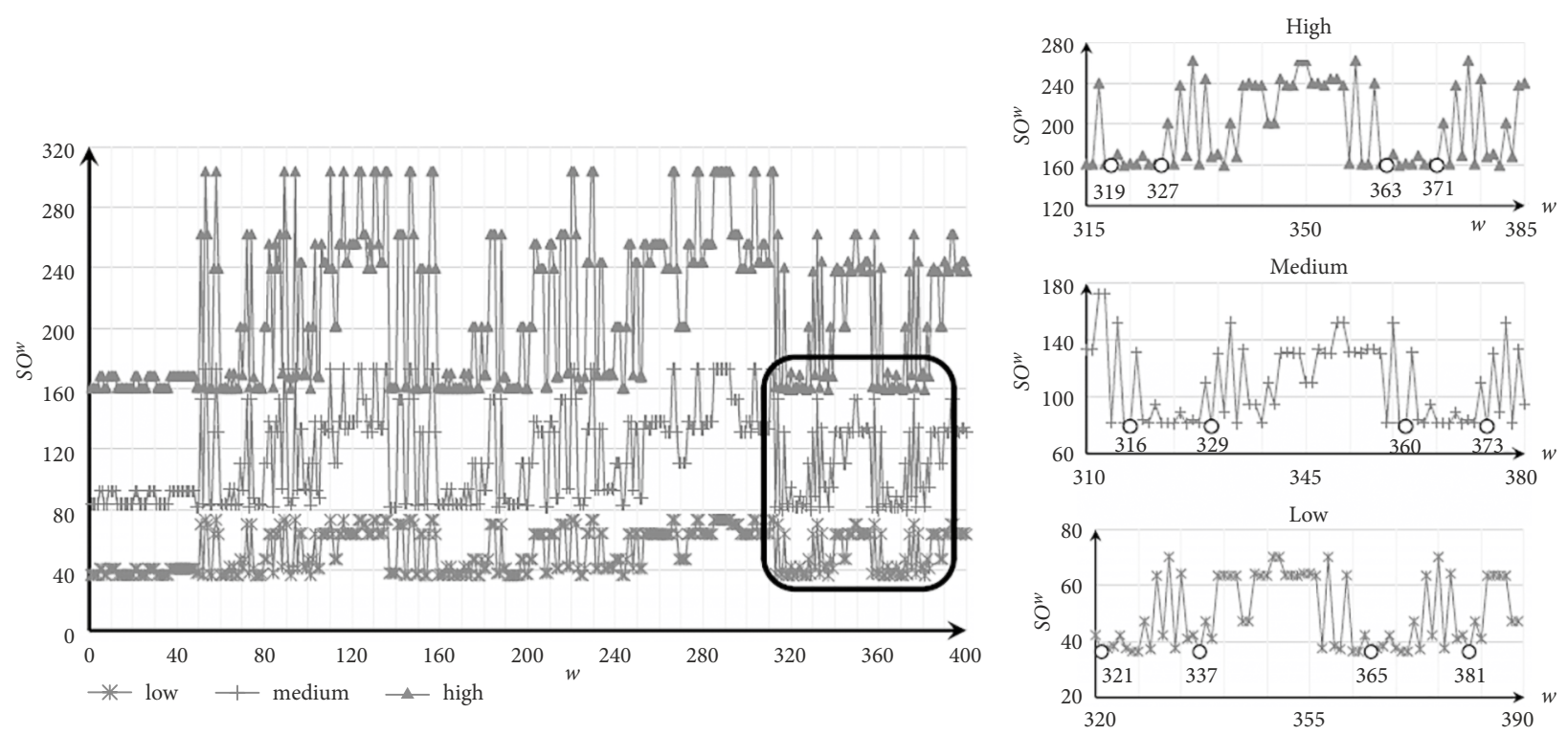

Figure 7. Optimization result of the proposal method

Table 8. Phase structure of conventional signal control schemes

\begin{tabular}{|l|c|l|c|}
\hline \multicolumn{1}{|c|}{$s$} & $w$ & \multicolumn{1}{|c|}{ PC schemes } & Phase \\
\hline DS & 1 & $\left\{\sigma_{11}, \sigma_{12}, \sigma_{13}\right\},\left\{\sigma_{21}, \sigma_{23}\right\},\left\{\sigma_{31}, \sigma_{33}\right\},\left\{\sigma_{41}, \sigma_{43}\right\}$ & 4 \\
\hline CS & 5 & $\left\{\sigma_{11}, \sigma_{12}, \sigma_{13}\right\},\left\{\sigma_{31}, \sigma_{33}\right\},\left\{\sigma_{21}, \sigma_{41}\right\},\left\{\sigma_{23}, \sigma_{43}\right\}$ & 4 \\
\hline RBS & 65 & $\left\{\sigma_{11}, \sigma_{12}, \sigma_{13}\right\},\left\{\sigma_{31}, \sigma_{33}\right\},\left\{\sigma_{21}, \sigma_{41}\right\},\left\{\sigma_{41}, \sigma_{43}\right\},\left\{\sigma_{23}, \sigma_{43}\right\}$ & 5 \\
\hline
\end{tabular}

Table 9. Results comparison of the objective values

\begin{tabular}{|l|c|c|c|c|c|c|c|}
\hline \multirow{2}{*}{$\begin{array}{c}\text { Demand } \\
\text { scenario }\end{array}$} & \multirow{2}{*}{$\mathrm{PM}$} & \multicolumn{2}{c|}{ DS } & \multicolumn{2}{c|}{ CS } & \multicolumn{2}{c|}{ RBS } \\
\cline { 3 - 8 } & & $S^{w}$ & increment & $S^{w}$ & increment & $S^{w}$ & Increment \\
\hline Low & 36.32680 & 36.78974 & 0.46294 & 40.8382 & 4.5114 & 36.63398 & 0.30718 \\
\hline Medium & 79.29897 & 83.48509 & 4.18612 & 91.97865 & 12.67968 & 83.07488 & 3.77591 \\
\hline High & 158.5066 & 159.7237 & 1.2171 & 167.6545 & 9.1479 & 159.3891 & 0.8825 \\
\hline
\end{tabular}

a)

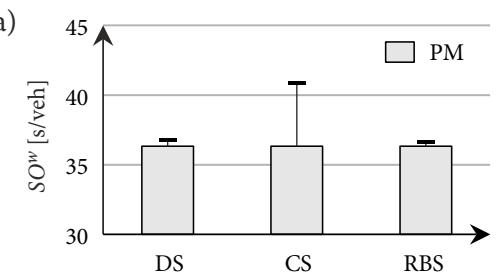

d)

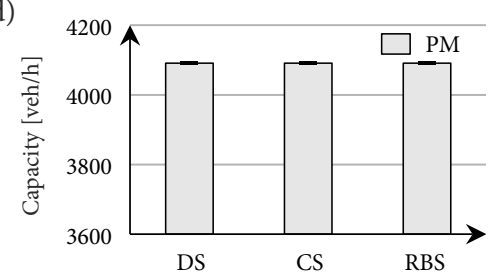

g)

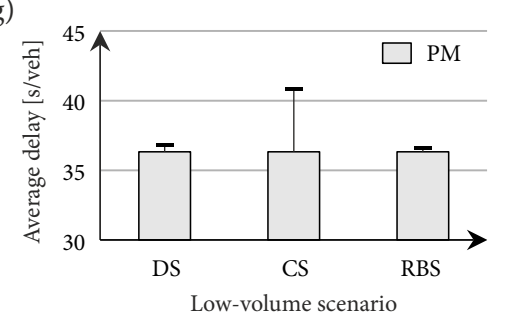

b)

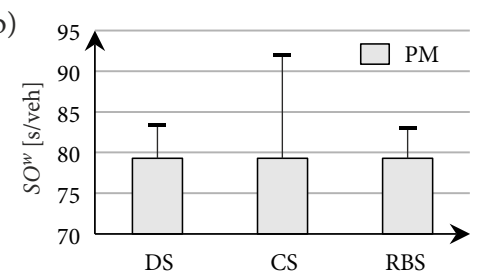

e)

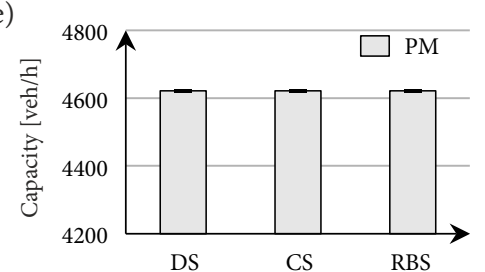

h)

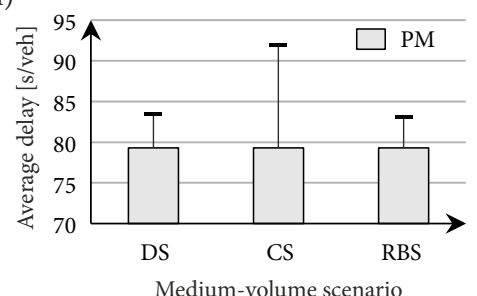

Figure 8. Performance evaluation results c)

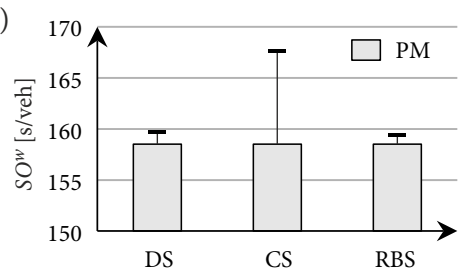

f)

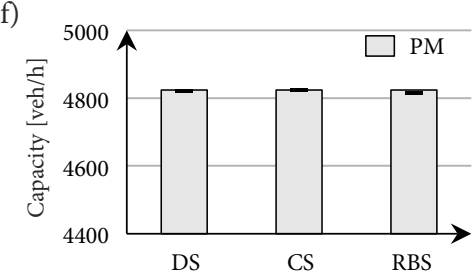

i)

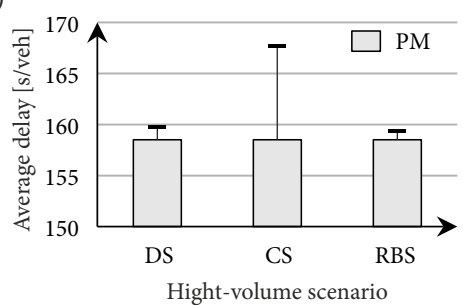


a)

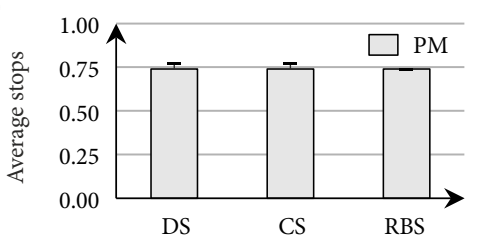

d)

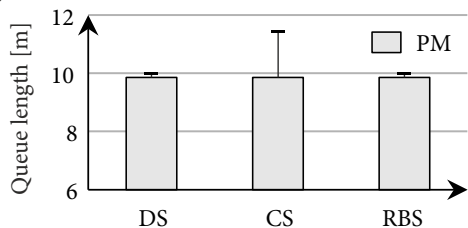

g)

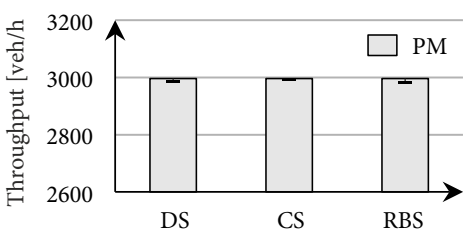

j)

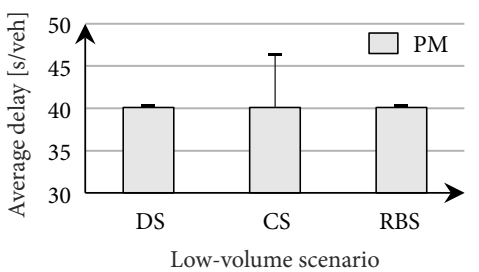

b)

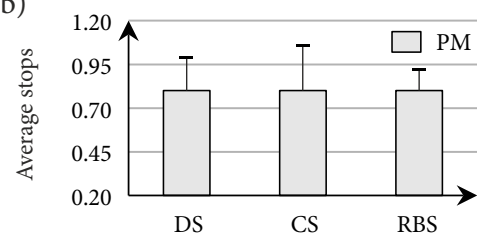

e)

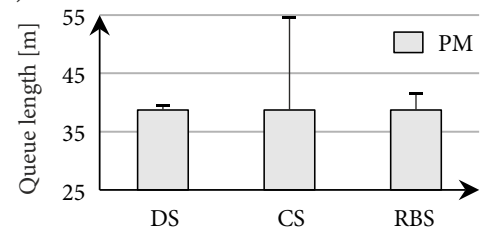

h)

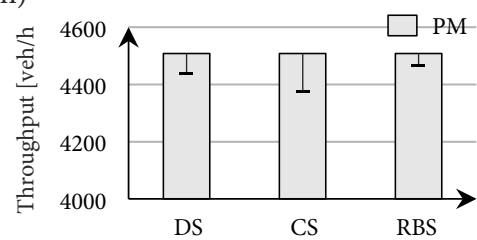

k)

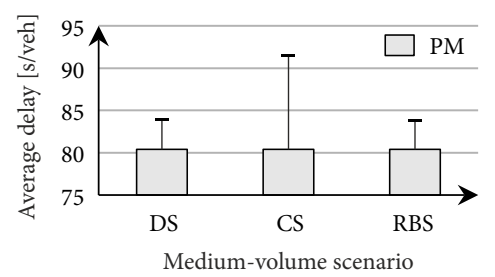

Figure 9. Simulation verification results

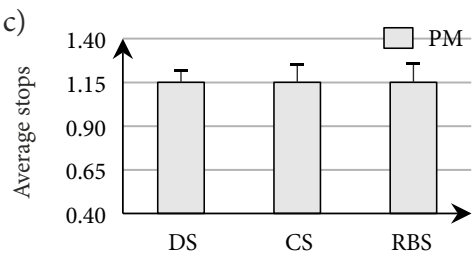

f)

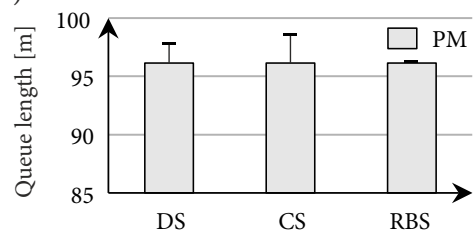

i)

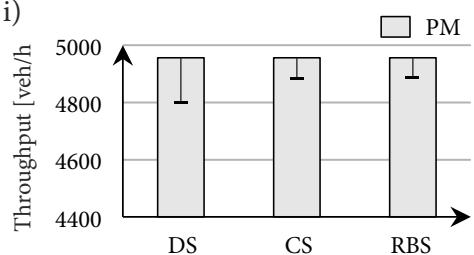

l)

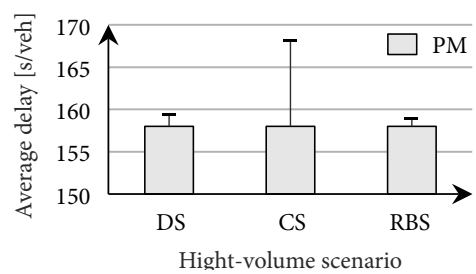

\section{Conclusions and further work}

A new optimal control scheme for intersections with considering the comprehensive styles of PMC under the automated vehicles environment was proposed in this paper. The proposed method considered the comprehensive PMC styles by fractionalizing movement compatibility relationships and use discrete mathematics to calculate overall FPC schemes according to the requirements of the signal phase. Then built the corresponding optimized timing model according to different PC schemes. To verify the feasibility and effectiveness of the proposed method, this paper introduced numerical analyses to verify these results. The analysis results showed that the proposed method can effectively reduce the average delay of vehicles at the intersection. The other following conclusions can be drawn from this research:

"» the PC scheme of the proposed method was more comprehensive than the conventional PC scheme. The number of PC scheme in this paper included a series of other combination schemes besides OS, DS, CS, and RBS (Table 5);

"» the different PC schemes of signalized intersections have significant influence on the intersection control effectiveness (Figure 7). The optimized objective function value was different because of the difference of PC schemes. The minimum and maximum target values were 36.3268 and $73.05468 \mathrm{~s} / \mathrm{veh}$, 79.29897 and 173.025 s/veh, 158.5066 and
$303.5569 \mathrm{~s} / \mathrm{veh}$, respectively for the low-, mediumand high-volume scenarios. The maximum and minimum target values were greater than or equal to 2 times the original values, which indicated that the signal PC selection had a significant influence on the control effectiveness of the signal phase scheme. Therefore, it was very important to select a reasonable PC scheme;

"» the control scheme had the same control effectiveness with the same PMC and different phase sequence. In Table 7, every scenario had four optimal control scheme, so it was not difficult to find that these schemes had the same PMC and different phase sequence;

"» compared with the conventional phase control scheme, the proposed method had a better control effectiveness. The numerical analyses showed that the overall objective values were improved, the traffic capacity was increased, and the average vehicle delay was further reduced than the conventional PC schemes (Table 9 and Figure 8). Especially under the Medium-volume scenario, the objective function value was smaller than CS by $12.67968 \mathrm{~s} / \mathrm{veh}$ and RBS by $3.77591 \mathrm{~s} / \mathrm{veh}$. The objective function result was the synthesis of the vehicle average delay and the intersection capacity, which can reflect the signal intersection optimization of the vehicle average delay and capacity. 
In conclusion, the proposed method in this paper improved the signal control effectiveness by optimizing the PMC of intersections, and provided a new idea for signal control optimization for the automated vehicles. However, the method considered only the compatibility relationship between the motorized vehicle movements and used the protection phase for left turn movements.

Future research will consider the optimization of signal control at intersections with the signal PC by increasing the pedestrian movements and non-motorized vehicle movements. In addition, the setting of the left turn permission phase should be considered to expand the application scope of the method. In addition, research on dynamic combination control of phase movements to fit the random achievement characteristics of movement vehicle.

\section{Acknowledgements}

The authors would like to acknowledge the support provided by the National Natural Science Foundation of China, and thank the anonymous reviewers for their invaluable comments.

\section{Funding}

This work was supported by the National Natural Science Foundation of China under Grant No U1664262.

\section{Author contributions}

Wenbin Xiao and Shunying Zhu conceived the study and were responsible for the review and analysis of the theories.

Daobin Wang was responsible for the experiment scheme and analysis.

Wei Liu was responsible for the data interpretation.

Wenbin Xiao wrote the first draft of the article.

\section{Disclosure statement}

We have no competing financial, professional, or personal interests from other parties.

\section{References}

Akçelik, R.; Rouphail, N. M. 1993. Estimation of delays at traffic signals for variable demand conditions, Transportation Research Part B: Methodological 27(2): 109-131. https://doi.org/10.1016/0191-2615(93)90003-S

Cai, J.; Liu, H. Y.; Zhang, L. H.; Wang, Z. 2013. The optimal unit green extension - considering different demand patterns, Applied Mechanics and Materials 409-410: 1357-1365. https://doi.org/10.4028/www.scientific.net/AMM.409-410. 1357

Chang, T.-H.; Sun, G.-Y. 2004. Modeling and optimization of an oversaturated signalized network, Transportation Research Part B: Methodological 38(8): 687-707. https://doi.org/10.1016/j.trb.2003.08.002
Comert, G. 2016. Queue length estimation from probe vehicles at isolated intersections: Estimators for primary parameters, European Journal of Operational Research 252(2): 502-521. https://doi.org/10.1016/j.ejor.2016.01.040

Feng, Y.; Head, K. L.; Khoshmagham, S.; Zamanipour, M. 2015. A real-time adaptive signal control in a connected vehicle environment, Transportation Research Part C: Emerging Technologies 55: 460-473. https://doi.org/10.1016/j.trc.2015.01.007

Fu, L.; Hellinga, B. 2000. Delay variability at signalized intersections, Transportation Research Record: Journal of the Transportation Research Board 1710: 215-221. https://doi.org/10.3141/1710-25

Gayah, V. V.; Daganzo, C. F. 2012. Analytical capacity comparison of one-way and two-way signalized street networks, Transportation Research Record: Journal of the Transportation Research Board 2301: 76-85. https://doi.org/10.3141/2301-09

Ghanbarikarekani, M.; Qu, X.; Zeibots, M.; Qi, W. 2018. Minimizing the average delay at intersections via presignals and speed control, Journal of Advanced Transportation 2018: 4121582. https://doi.org/10.1155/2018/4121582

Goldblatt, R.; Mier, F.; Friedman, J. 1994. Continuous flow intersections, ITE Journal 64(7): 35-42.

Guler, S. I.; Cassidy, M. J. 2012. Strategies for sharing bottleneck capacity among buses and cars, Transportation Research Part B: Methodological 46(10): 1334-1345. https://doi.org/10.1016/j.trb.2012.09.002

Hao, P.; Ban, X.; Bennett, K. P.; Ji, Q.; Sun, Z. 2012. Signal timing estimation using sample intersection travel times, IEEE Transactions on Intelligent Transportation Systems 13(2): 792804. https://doi.org/10.1109/TITS.2012.2187895

He, Q.; Head, K. L.; Ding, J. 2014. Multi-modal traffic signal control with priority, signal actuation and coordination, Transportation Research Part C: Emerging Technologies 46: 65-82. https://doi.org/10.1016/j.trc.2014.05.001

Hummer, J. E. 1998a. Unconventional left-turn alternatives for urban and suburban arterials - part one, ITE Journal 68(9): $26-29$.

Hummer, J. E. 1998b. Unconventional left-turn alternatives for urban and suburban arterials - part two, ITE Journal 68(11): 101-106.

Jamson, A. H.; Merat, N.; Carsten, O. M. J.; Lai, F. C. H. 2013. Behavioural changes in drivers experiencing highly-automated vehicle control in varying traffic conditions, Transportation Research Part C: Emerging Technologies 30: 116-125. https://doi.org/10.1016/j.trc.2013.02.008

Klanšek, U. 2015. A comparison between MILP and MINLP approaches to optimal solution of nonlinear discrete transportation problem, Transport 30(2): 135-144.

https://doi.org/10.3846/16484142.2014.933361

Lee, J.; Park, B. 2012. Development and evaluation of a cooperative vehicle intersection control algorithm under the connected vehicles environment, IEEE Transactions on Intelligent Transportation Systems 13(1): 81-90. https://doi.org/10.1109/tits.2011.2178836

Li, Y.; Yu, L.; Tao, S.; Chen, K. 2013. Multi-objective optimization of traffic signal timing for oversaturated intersection, Mathematical Problems in Engineering 2013: 182643. https://doi.org/10.1155/2013/182643

Li, Z.; Elefteriadou, L.; Ranka, S. 2014. Signal control optimization for automated vehicles at isolated signalized intersections, Transportation Research Part C: Emerging Technologies 49: 1-18. https://doi.org/10.1016/j.trc.2014.10.001 
Liu, H. X.; Wu, X.; Ma, W.; Hu, H. 2009. Real-time queue length estimation for congested signalized intersections, Transportation Research Part C: Emerging Technologies 17(4): 412-427. https://doi.org/10.1016/j.trc.2009.02.003

Liu, X.; Benekohal, R. F.; Shaik, M. A. R. 2017. Queue length at signalized intersections from red-time formula and the Highway Capacity Manual compared with field data, Transportation Research Record: Journal of the Transportation Research Board 2615: 159-168. https://doi.org/10.3141/2615-18

Liu, Y.; Chang, G.-L. 2011. An arterial signal optimization model for intersections experiencing queue spillback and lane blockage, Transportation Research Part C: Emerging Technologies 19(1): 130-144. https://doi.org/10.1016/j.trc.2010.04.005

Lucas, D. E.; Mirchandani, P. B.; Head, K. L. 2000. Remote simulation to evaluate real-time traffic control strategies, Transportation Research Record: Journal of the Transportation Research Board 1727: 95-100. https://doi.org/10.3141/1727-12

Mung, G. K. S.; Poon, A. C. K.; Lam, W. H. K. 1996. Distributions of queue lengths at fixed time traffic signals, Transportation Research Part B: Methodological 30(6): 421-439. https://doi.org/10.1016/0191-2615(96)00009-4

Suh, W.; Hunter, M. P. 2014. Signal design for displaced leftturn intersection using Monte Carlo method, KSCE Journal of Civil Engineering 18(4): 1140-1149. https://doi.org/10.1007/s12205-014-0225-8

Sun, D.; Benekohal, R. F.; Waller, S. T. 2006. Bi-level programming formulation and heuristic solution approach for dynamic traffic signal optimization, Computer-Aided Civil and Infrastructure Engineering 21(5): 321-333. https://doi.org/10.1111/j.1467-8667.2006.00439.x

TRB. 2010. Highway Capacity Manual. Transportation Research Board (TRB), Washington DC, US. 1650 p.

Wong, C. K.; Heydecker, B. G. 2011. Optimal allocation of turns to lanes at an isolated signal-controlled junction, Transportation Research Part B: Methodological 45(4): 667-681. https://doi.org/10.1016/j.trb.2010.12.001

Wong, C. K.; Wong, S. C. 2003. Lane-based optimization of signal timings for isolated junctions, Transportation Research Part B: Methodological 37(1): 63-84. https://doi.org/10.1016/S0191-2615(01)00045-5

Wu, N.; Giuliani, S. 2016. Capacity and delay estimation at signalized intersections under unsaturated flow condition based on cycle overflow probability, Transportation Research Procedia 15: 63-74. https://doi.org/10.1016/j.trpro.2016.06.006

Xuan, Y.; Daganzo, C. F.; Cassidy, M. J. 2011. Increasing the capacity of signalized intersections with separate left turn phases, Transportation Research Part B: Methodological 45(5): 769-781. https://doi.org/10.1016/j.trb.2011.02.009

Yang, Q.; Shi, Z. 2017. Performance analysis of the phase swap sorting strategy for an isolated intersection, Transportation Research Part C: Emerging Technologies 77: 366-388. https://doi.org/10.1016/j.trc.2017.01.018

Zhang, G.; Wang, Y. 2011. Optimizing minimum and maximum green time settings for traffic actuated control at isolated intersections, IEEE Transactions on Intelligent Transportation Systems 12(1): 164-173. https://doi.org/10.1109/tits.2010.2070795

Zhao, J.; Ma, W.; Zhang, H. M.; Yang, X. 2013. Increasing the capacity of signalized intersections with dynamic use of exit lanes for left-turn traffic, Transportation Research Record: Journal of the Transportation Research Board 2355: 49-59. https://doi.org/10.3141/2355-06
Zhao, J.; Ma, W.; Head, K. L.; Yang, X. 2015a. Optimal operation of displaced left-turn intersections: a lane-based approach, Transportation Research Part C: Emerging Technologies 61: 29-48. https://doi.org/10.1016/j.trc.2015.10.012

Zhao, S.; Liang, S.; Liu, H.; Ma, M. 2015b. CTM based real-time queue length estimation at signalized intersection, Mathematical Problems in Engineering 2015: 328712. https://doi.org/10.1155/2015/328712

Zheng, X.; Recker, W.; Chu, L. 2010. Optimization of control parameters for adaptive traffic-actuated signal control, Journal of Intelligent Transportation Systems: Technology, Planning, and Operations 14(2): 95-108.

https://doi.org/10.1080/15472451003719756 